\title{
In Praise and in Criticism of the Model of Continuous Spontaneous Localization of the Wave-Function
}

\author{
Sofia D. Wechsler \\ Kiryat Motzkin, Israel \\ Email: sofia10@012.net.il
}

How to cite this paper: Wechsler, S.D. (2020) In Praise and in Criticism of the Model of Continuous Spontaneous Localization of the Wave-Function. Journal of Quantum Information Science, 10, 73-103. https://doi.org/10.4236/jqis.2020.104006

Received: August 8, 2020

Accepted: October 27, 2020

Published: October 30, 2020

Copyright $\odot 2020$ by author(s) and Scientific Research Publishing Inc. This work is licensed under the Creative Commons Attribution International License (CC BY 4.0).

http://creativecommons.org/licenses/by/4.0/

\begin{abstract}
Different attempts to solve the measurement problem of the quantum mechanics $(\mathrm{QM})$ by denying the collapse principle, and replacing it with changes in the quantum formalism, failed because the changes in the formalism lead to contradictions with QM predictions. To the difference, Ghirardi, Rimini and Weber took the collapse as a real phenomenon, and proposed a calculus by which the wave-function should undergo a sudden localization. Later on, Ghirardi, Pearle and Rimini came with a change of this calculus into the CSL (continuous spontaneous localization) model of collapse. Both these proposals rely on the experimental fact that the reduction of the wave-function occurs when the microscopic system encounters a macroscopic object and involves a big amount of its particles. Both these proposals also change the quantum formalism by introducing in the Schrödinger equation additional terms with noisy behavior. However, these terms have practically no influence as long as the studied system contains only one or a few components. Only when the amount of components is very big, these terms become significant and lead to the reduction of the wave-function to one of its components. The present work has two purposes: 1) proving that the collapse postulate is unavoidable; 2) applying the CSL model to the process in a detector and showing step by step the modification of the wave-function, until reduction. As a side detail, it is argued here that the noise cannot originate in some classical field, contrary to the thought/hope of some physicists, because no classical field is tailored by the wave-functions of entanglements.
\end{abstract}

\section{Keywords}

Quantum Mechanics, Collapse 


\section{Introduction}

In a profound analysis of tests of quantum systems, [1], J. von Neumann concluded that once a quantum system in the initial state $\psi$ is tested (non-destructively) and produces the result $\lambda_{k}$, the system remains in a state $\phi_{k}$ with the property that any subsequent measurement of the system for the same observable, would produce the same result, $\lambda_{k}$ (see for instance page 138 in [1]). This conclusion was always confirmed by the experiment. G. Lüders refined von Neumann's work examining cases with degenerate eigenvalues [2]. But the question remains what happens with the other components $\phi_{1}, \cdots, \phi_{k-1}, \phi_{k+1}, \cdots, \phi_{N}$ of the initial wave-function. Do they disappear, or do they continue to exist?

The complementary question concerns tests done exclusively for the result $\lambda_{k}$, in which the detector remains silent. For instance, in the test of a one particle wave-function (OPWF) comprising $N$ wave-packets space-separated, a detector is placed only on the wave-packet $\phi_{k}$. If the detector does not click, was $\phi_{k}$ destroyed?

Some scientists believe that $\phi_{1}, \cdots, \phi_{k-1}, \phi_{k+1}, \cdots, \phi_{N}$ in the first case, and $\phi_{k}$ in the second case, disappear. But neither von Neumann, nor Lüders, brought a rigorous proof that such a disappearance really occurs. So, the reduction of the wave-function, or "collapse”, remained just as a postulate. Lüders motivated:

"statements on the change of state due to measurement do not arise out of quantum theory itself through the inclusion of the measurement apparatus in the Schrödinger equation. Measurement, an act of cognizance, adds an element not already contained in the formulation of quantum theory."

Other physicists, displeased by the enigmatic collapse postulate, launched "interpretations" of the quantum mechanics (QM). Trying to explain the process of measurement of quantum systems without this postulate, they introduced modifications in the standard quantum formalism, or even additional universes. The price of altering the formalism was a contradiction with the quantum predictions for one or another experiment. So happened with the most popular interpretations, e.g. the mechanics of de Broglie and Bohm [3] $[4],{ }^{1}$ the full/empty waves hypothesis-see for example [6] for explanation of the concept ${ }^{2}$ - the consistent histories [7], ${ }^{3}$ the transactional interpretation

\footnotetext{
${ }^{1}$ The de Broglie-Bohm interpretation of QM is based on the assumption of particles floating inside the wave-function and following continuous trajectories. The existence of such continuous trajectories was disproved in [5] Section 3.

${ }^{2}$ From the proof against continuous trajectories in the Section 3 of [5] one infers that a "full wave" cannot follow a continuous trajectory. On the other hand, neither can it jump from one region to another one, space-separated, because in this case a wave-packet could be an empty wave when meeting a detector, and become a full wave later and trigger a subsequent detector. That would contradict our experiments on quantum systems.

${ }^{3}$ The consistent histories theory is mainly due to R. Griffith. Regrettably, it disobeys the quantum formalism. As an example, in the "histories" (13.7) in [7] it appears that after passing through a beam-splitter, the wave-function is truncated. Such a truncation is not allowed by the quantum formalism, since the transformation of the wave-function by a beam-splitter is unitary.
} 
$[8] .^{4}$

All these interpretations ignored the well known experimental fact that the reduction of the wave-function occurs in the presence of a macroscopic object and perturbs so many of its particles until its macroscopic state changes.

There is one proposal which, to the difference from the above interpretations, took the collapse postulate "seriously", and suggested a bridge between the quantum and the classical formalism. Ghirardi, Rimini and Weber (GRW) [9] thought that the wave-function of a quantum system might undergo at random times a sudden shrinking to a small region (localization). They too proposed changes in the Schrödinger equation; however, the changes have negligible effect on the quantum system containing a small number of components, and great effect-localization-when very many components are involved. Thus, in fact, the quantum formalism would not be changed.

A few years later, Ghirardi, Pearle, and Rimini (GPR) came with a refined version of the GRW proposal, the "continuous spontaneous localization" (CSL) $[10],{ }^{5}$ by which the localization occurs progressively in time, instead of suddenly. ${ }^{6}$

The modification in the Schrödinger equation consists in adding a stochastic noise and non-linear terms. The stochasticity of the noise mimics the stochasticity of the result of the measurement. The magnitude of these supplementary terms increases with the number of particles involved.

The issue that the collapse occurs in the presence of many particles was put by D. Bedingham in a definite way, [17]

"Our experience in the use of quantum theory tells us that the state reduction postulate should not be applied to a microscopic system consisting of a few elementary particles until it interacts with a macroscopic object such as a measuring device."

Remark 1: This text uses frequently the word "particle", and it also appears in citations. Unless otherwise specified, this word means a simple quantum system, of one or a few components.

R. Feynman also described the effect of the involvement of a big amount of particles-section 2.3 of [18],

${ }^{4}$ Inspired by the Wheeler-Feynman absorber theory, J. G. Crammer proposed the hypothesis that both the emitter of a quantum system and the detector, emit a forward-in-time wave and a backward-in-time wave. The detection is supposed to occur if "hand-shake" occurs between the forward-in-time wave of the emitter and the backward-in-time wave of the detector. However, to the difference from the Wheeler-Feynman absorber theory in which the two waves superpose, in the formalism of the transaction interpretation appears the arithmetical product of the two waves. This replacement is motivated by claiming that the product gives the Born rule. But that is at variance with the quantum formalism in which the Born rule involves the inner product (an integration over the variables) of two waves, not their simple arithmetical product.

${ }^{5}$ In fact, the model in [10] is a continuation and enhancement of proposals of N. Gisin, [11], and of Pearle [12].

${ }^{6}$ This model is described also in the Section 3 of [13], Sections 7 and 8 of [14], section II F in [15], and section II C of [16]. 
"The classical approximation, however, corresponds to the case that the dimensions, masses, times, etc., are so large that $S$ is enormous in relation to $\hbar\left(=1.05 \times 10^{-27} \mathrm{erg} \cdot \mathrm{sec}\right)$. Then the phase of the contribution $\mathrm{S} / \hbar$ is some very, very large angle ... small changes of path will, generally, make enormous changes in phase, ... The total contribution will then add to zero; for if one path makes a positive contribution, another infinitesimally close (on a classical scale) makes an equal negative contribution. ... But for the special path $\bar{x}(t)$, for which $S$ is an extremum, a small change in the path produces, in the first order at least, no change in S. All the contributions from the paths in this region are nearly in phase, ..., and do not cancel out"

So, when the number of components of a system increases so much that it becomes a macroscopic object, the wave-function of the total system is destroyed. Unfortunately, Feynman's explanation covers only the particular case of a OPWF consisting in a single wave-packet, not in a superposition of a couple of wave-packets space-separated.

The present work focuses on the CSL formalism. This formalism is not regarded as an explanation of the collapse process, because it is not yet known which field is this noisy field. The stochastic Schrödinger equation (SSE) is regarded as the best tool for investigating the collapse, and what this text does is to apply the CSL model for following what happens in a detector during the detector process. None of the "interpretations" of QM is able to do such a thing.

Applied to the process in a detector, the model predicts the expected predictions: as long as a small number of particles from the detector are entrained, no localization occurs. However, as this number increases the localization appears. It is dictated by the noise. Some evolutions of the noise enhance very much the number of involved particles and the detector clicks. Other evolutions of the noise begin, at a certain time, to reduce the number of involved particles, and in the end the detector remains unperturbed and does not click; also, the wave-packet which did not trigger the detector, is erased.

Another purpose of this work is to prove that, in partial disagreement with Lüders, the disappearance of a wave-packet that didn't trigger a detector placed on its path, is demanded by the QM formalism. It is strange that the supporters of the CSL model didn't try to prove that. The present work tries to remove this lacuna.

By the end of writing this work I was notified of a recent article, [19], which also studies the detection process with the CSL model, though, with a different approach and a more complicated setup. To the difference from the present work, which shows step by step the wave-function reduction as the process in a detector unfolds, in [19] is examined the process in another element of the electric circuit, completely classical, which does not come in contact with the wave-function. A brief description of the analysis in [19] is done in Subsection 5.5 .

This mysterious noise in the SSE challenged the supporters of the CSL model to ask which field might stand behind it. N. Gisin pointed out in [20] that the 
interaction appearing in SSE between the quantum system and this noise is similar with the interaction of a quantum system with an environment. L. Diósi advanced the idea that it may be a universal gravitational noise [21] [22] [23], but Ghirardi et al. criticized that proposal [24].

Personally, I don't believe that the noisy field may be classical, despite the hope expressed by Bassi and Ghirardi in [25], that the noise would be ultimately proved to be classical, e.g. gravitational. Although the present work does not deal with entanglements, it can be said in general that a classical field and its noise cannot be tailored in each type of experiment by the respective wave-function. Even within a single type of experiment, the noise won't be tailored by the setup that each experimenter chooses for his/her particle, all the more that each choice is at the free will of the chooser. Worse than that, the requirements on the noise are bound to become harder if the experimenters' labs are in relative movement.

The rest of the text is organized as follows: Section 2 describes the evolution of the detection in a macroscopic detector, taking as an example a proportional counter with gas ionization. Section 3 proves a couple of theorems in support of the necessity of the reduction postulate. Section 4 develops the mathematical tools for the treatment of a stochastic process, starting from the general Itô equation. Then it applies the tools on a microscopic system, showing that, in agreement with the principles of the CSL model, such a system won't undergo localization. Section 5 treats the detection process in a gas proportional counter. It is proved that as the number of the electron-ion pairs produced by ionization increases, the localization occurs. A difficulty is pointed to, which remains to be examined in future works. Section 6 contains conclusions.

\section{The Macroscopic Detector}

Typically, a macroscopic detector contains a sensitive material. When some particle enters the detector, some physical property of the respective type of particle interacts with the sensitive material producing a perturbation which is amplified in different ways, and a detection is reported. Or, if the wave-function consists in a quantum superposition and a detector is placed only on one of the wave-packets, in part of the trials of the experiment the detector remains silent.

In this text the proportional counter of cylindrical symmetry is taken as example of detector, Figure 1. The detailed description of this apparatus and its functioning can be found in [26] [27] [28].

A gas, usually of the noble type as $\mathrm{He}, \mathrm{Ar}, \mathrm{Xe}$, or others, is placed between two electrodes of opposite electrical charges. The incident particle ionizes a couple of atoms in the gas generating so-called "primary electrons and ions".

Both the electrons and the ions are accelerated by the electric field, though the electrons, having the mass at least $10^{4}$ times smaller than an ion, get a correspondingly higher acceleration. Thus, within the time necessary to the electron for reaching the anode, the ion practically doesn't move.

In the cylindrical counter the anode is a thin metallic wire passing through the 


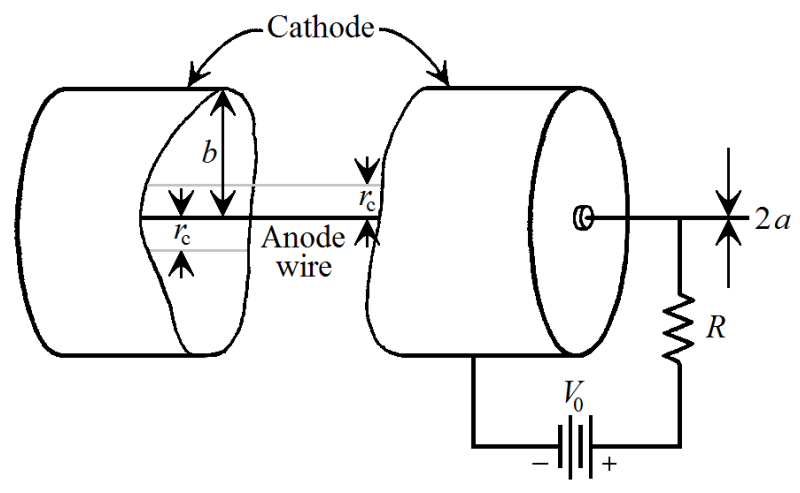

Figure 1. A proportional counter of cylindrical symmetry. (See explanations in the text).

center of the tube. Thus, the electric field increases as the distance from the anode decreases. At a certain distance $r_{\mathrm{c}}$ from the central axis the field becomes so intense that the "primary electrons" mentioned above have gained enough high kinetic energy for ionizing other atoms-Figure 2(a). Thus, the number of free electrons is doubled. The free electrons available after this first generation of so-called "secondary ionizations", are quickly accelerated and undergo a second generation of pair-producing collisions (PPCs), doubling again the number of free electrons.

The process continues this way, each primary electron producing in short time an "avalanche"-Figure 2(b) - named in the literature "Townsend avalanche", and the number of secondary electrons per primary electron is called multiplication factor. The region $r<r_{\mathrm{c}}$ is called the "avalanche region".

After a certain amount of pairs are produced, the (almost) static cloud of ions in the avalanche region limits the intensity of the field, so, the production of additional pairs is stopped. The electrons produced so far are absorbed by the anode, entailing a short output signal with steep rise. The positive ions drift slowly toward the cathode producing an additional signal, much longer and with slower rise.

Some atoms may absorb the energy of the hitting electrons, but instead of undergoing ionization they rise to upper levels from which they de-excite with emission of energetic photons. Such a photon interacts further with an atom and sets free an electron by the photo-electric effect. The electron starts an additional avalanche, in the same way as a primary electron did. However, in a proportional counter such a process has negligible probability.

\section{The Collapse Principle Is Unavoidable}

The purpose of the present section is to prove that the principle of wave-function reduction is unavoidable. We assume that the detectors are ideal. We will consider an OPWF with a couple of wave-packets, and for simplicity we will restrict our study to the case in which only one of the wave-packets illuminates a detector. One of two events will occur: the detector clicks, or, it "responds" by silence. 


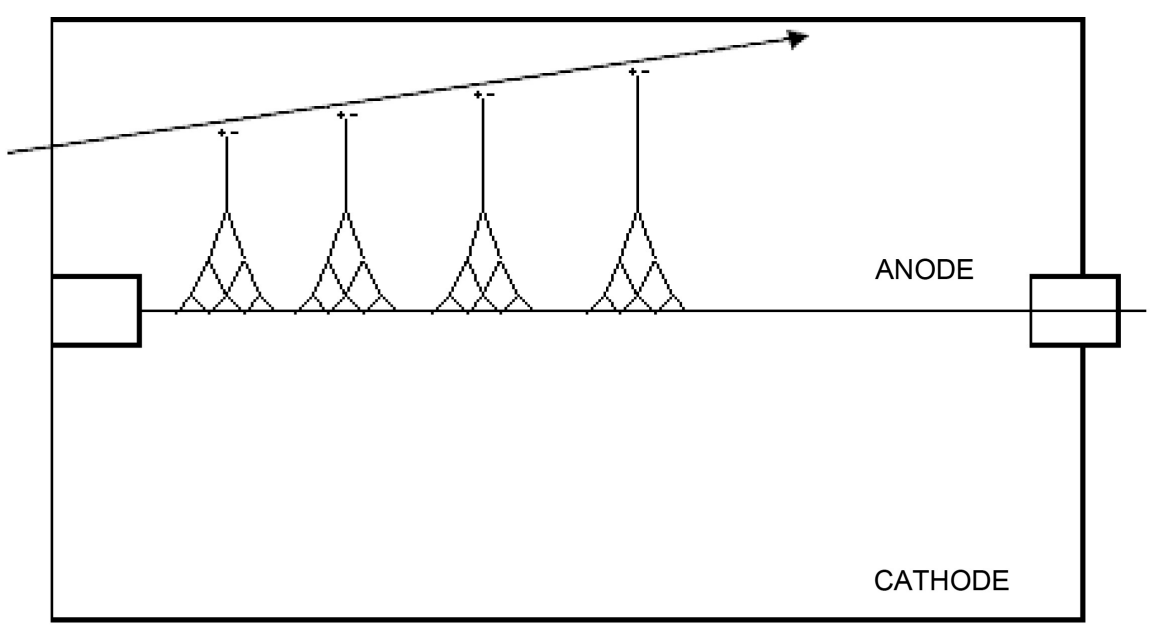

(a)

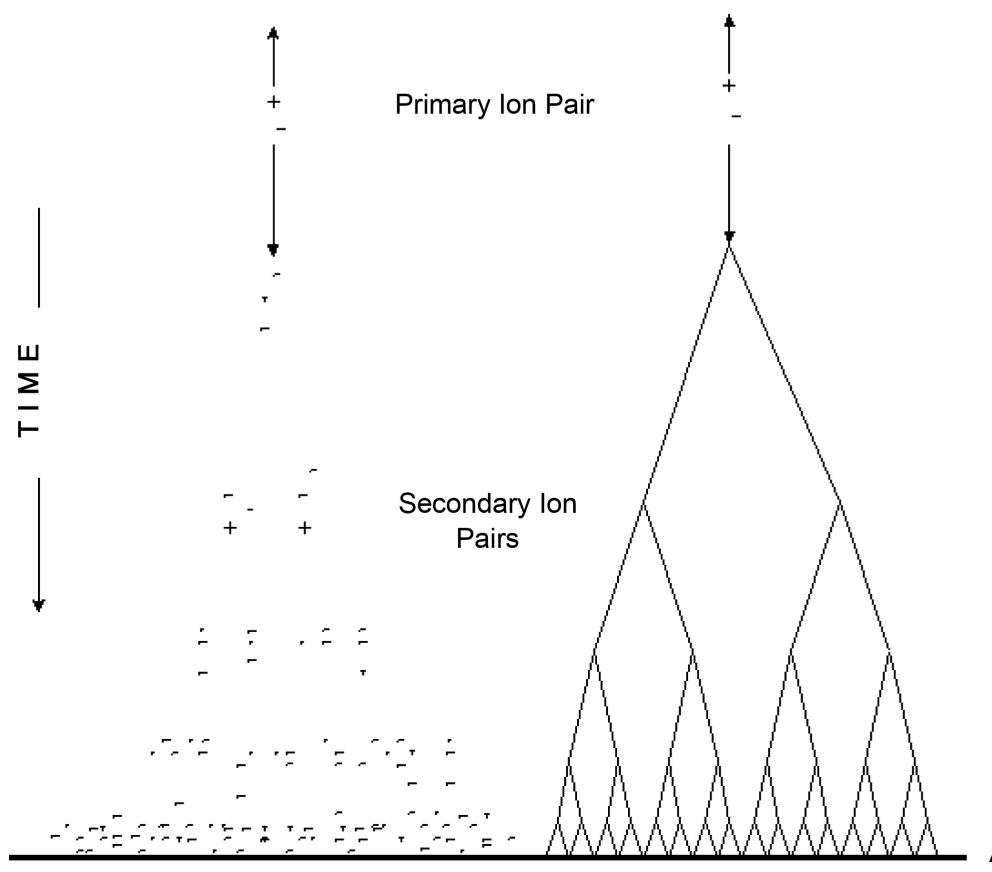

ANODE (+)

(b)

Figure 2. Amplification in proportional detectors. Both figures (a) and (b) are taken from the reference [27]. (a) An energetic incident particle collides with the atoms in the counter, ionizing some of them. The electrons resulting from the direct interaction of the visiting particle with the atoms move toward the anode, and in the avalanche region $r<r_{\text {c }}$ their energy increases so much that they ionize additional atoms, producing additional electron-ion pairs. The electrons are accelerated until another generation of ionizations occurs. (b) The process continues in the same way, each generation of ionizations doubling the number of free electrons. After a couple of such generations, whole avalanches are created.

\subsection{Measurements in Which the Detector Remains Silent}

How could a detector remain non-impressed by a wave-packet?

Many scenarios can be proposed. It is impossible to deal with all the suggestions the imagination can advance, all the more that some suggestions may con- 
tradict in different ways the laws of physics. There are two options which seem to me plausible:

1) The perturbation produced by the wave-packet in the detector is too small for changing the macroscopic state of the material in the detector.

2) The wave-packet is retro-flected in the detector, and exits without perturbing even one particle.

The following theorems rule out these options.

\section{Theorem 1:}

If a wave-packet meets a detector without triggering it, no particle in the detector remains perturbed.

\section{Proof.}

From a down-conversion pair of photons, the idler photon is sent to a detector $\mathrm{Q}$, and the signal photon is sent to a detector $\mathrm{S}$-Figure 3. The signal wave-packet is split by the $50 \%-50 \%$ beam-splitter BS, into two copies, one reflected, $|a\rangle$, and one transmitted, $|b\rangle$. Both copies travel to a rotating mirror $\mathrm{M}$, initially in horizontal position. The paths from the nonlinear crystal which produces the pair (not shown in the figure) to the detector $\mathrm{Q}$ and to the mirror $\mathrm{M}$ are tuned so that the idler reaches and triggers $\mathrm{Q}$, just after the wave-packet $|a\rangle$ was reflected by $\mathrm{M}$. Upon the click of $\mathrm{Q}, \mathrm{M}$ is rotated to vertical position. Thus, the wave-packet $|b\rangle$ which reaches the region of the mirror $M$ later than $|a\rangle$ because of a retarding system of mirrors $\mathrm{m}$, does not meet $\mathrm{M}$, and continues its travel on the same track as $|a\rangle$. At this step the wave-function of the signal photon becomes, considering all the reflections at mirrors,

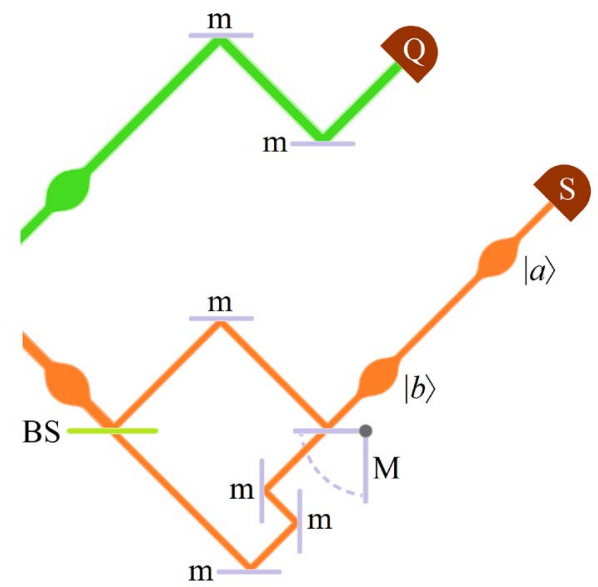

Figure 3. A which-way experiment. The colors in the figure are only for eye-guiding. BS is a beam-splitter, $\mathrm{m}$ are fixed mirrors, $\mathrm{M}$ is a rotatable mirror, $\mathrm{S}$ and $\mathrm{Q}$ are ideal detectors. From a down-conversion pair, the idler photon (green) travels toward the detector $\mathrm{Q}$, and the signal photon (orange) lands on a fair beam-splitter BS, where its wave-packet is split into a reflected part $|a\rangle$, and a transmitted part $|b\rangle$. The mirror $\mathrm{M}$ is initially in horizontal position, thus, $|a\rangle$ is reflected by $\mathrm{M}$ and directed toward the detector S. Immediately after that, the idler photon meets the detector $\mathrm{Q}$ which clicks. Upon this event, $\mathrm{M}$ is rotated to vertical. So, the wave-packet $|b\rangle$ retarded by three mirrors $\mathrm{m}$, doesn't en- 
counter the mirror M and travels towards the detector S, along the same track as $|a\rangle$.

$$
|\phi\rangle=-(|a\rangle+\mathrm{i}|b\rangle) / \sqrt{2} \text {. }
$$

In continuation, the wave-packets $|a\rangle$ and $|b\rangle$ follow their common track toward the detector S. The wave-packet $|a\rangle$ is the first one to meet the detector. At the interaction with a first particle (atom/molecule) from the sensitive material in the detector, an entanglement is generated

$$
|\phi\rangle\left|A_{1}^{\mathrm{u}}\right\rangle \rightarrow-\left(\left|a^{\mathrm{p}}\right\rangle\left|A_{1}^{\mathrm{p}}\right\rangle+\mathrm{i}\left|b^{\mathrm{u}}\right\rangle\left|A_{1}^{\mathrm{u}}\right\rangle\right) / \sqrt{2},
$$

where the upper-script "p" means "perturbed", and "u" means "unperturbed".

Both the incoming particle and the one belonging to the detector are perturbed. Further, they meet and perturb additional particles as explained in the previous section. As long as the number of perturbed particles is small, their total system continues to be described by the quantum formalism,

$$
|\Phi\rangle=-\left(\left|a^{\mathrm{p}}\right\rangle\left|A_{1}^{\mathrm{p}}\right\rangle\left|A_{2}^{\mathrm{p}}\right\rangle \cdots+\mathrm{i}\left|b^{\mathrm{u}}\right\rangle\left|A_{1}^{\mathrm{u}}\right\rangle\left|A_{2}^{\mathrm{u}}\right\rangle \cdots\right) / \sqrt{2}
$$

Assume that meanwhile, the wave-packet $|b\rangle$ also reaches the detector S. It starts a new chain of perturbations. For simplicity, we assume that the two sets of perturbed particles are disjoint.

Let $t$ be a time at which $|a\rangle$ engaged $N$ perturbed particles, and $|b\rangle, M$ perturbed particles, with both $N$ and $M$ still small enough for allowing a quantum description. Equation (3) evolved into

$$
|\Theta\rangle=-\left(\left|a^{\mathrm{p}}\right\rangle\left|A_{1}^{\mathrm{p}}\right\rangle \cdots\left|A_{N}^{\mathrm{p}}\right\rangle\left|A_{1}^{\prime \mathrm{u}}\right\rangle \cdots\left|A_{M}^{\prime \mathrm{u}}\right\rangle+\mathrm{i}\left|b^{\mathrm{p}}\right\rangle\left|A_{1}^{\mathrm{u}}\right\rangle \cdots\left|A_{N}^{\mathrm{u}}\right\rangle\left|A_{1}^{\prime \mathrm{p}}\right\rangle \cdots\left|A_{M}^{\prime \mathrm{p}}\right\rangle\right) / \sqrt{2} .
$$

The particles correlated with $|b\rangle$ were marked with a prime for avoiding confuse with those correlated with $|a\rangle$.

Now, let's see what the RHS of (4) tells us.

One can see that if the particles $A_{1}^{\prime} \cdots A_{M}^{\prime}$ are perturbed, then $A_{1} \cdots A_{N}$ should not have been perturbed, therefore they could not begin an avalanche. In particular, one can set $N=1$.

Symmetrically, if the particles $A_{1} \cdots A_{N}$ were perturbed, then $A_{1}^{\prime} \cdots A_{M}^{\prime}$ are forced to remain unperturbed, therefore they cannot begin an avalanche. In particular, one can set $M=1$.

That confirms the theorem and rules out the option (1).

\section{Theorem 2:}

$A$ wave-packet that did not impress an ideal, absorbing detector, was destroyed.

\section{Proof:}

As the ideal detector has perfectly reflecting walls, the escape can be only by retro-flection on the walls and exit through the input window.

Consider again a pair of down-conversion photons, in which the idler is sent to a detector Q, and the signal to a beam-splitter BS-Figure 4. At BS, the signal wave-packet is split into a reflected copy $|a\rangle$, and a transmitted copy $|b\rangle$. The latter flies in continuation through the vicinity of a rotating mirror $M$, initially 


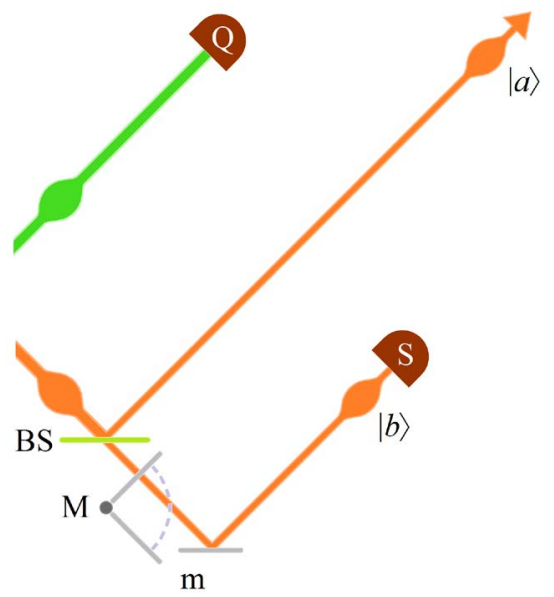

Figure 4. Test of the possibility of retro-flection of a wave-packet by an ideal detector. The colors in the figure are only for eye-guiding. BS is a beam-splitter, $\mathrm{m}$ is a fixed mirror, $\mathrm{M}$ is a rotatable mirror, $\mathrm{S}$ and $\mathrm{Q}$ are ideal, absorbing detectors. From a down-conversion pair, the idler photon (green) travels toward the detector $\mathrm{Q}$, and the signal photon (orange) to BS. The mirror M is initially parallel to the path of the transmitted beam $|b\rangle$, therefore the beam does not meet the mirror. Then, $|b\rangle$ travels on and is reflected by the fixed mirror $\mathrm{m}$. At this time, the idler photon meets the detector $\mathrm{Q}$ which clicks. Upon the click of $\mathrm{Q}, \mathrm{M}$ is rotated to a position perpendicular to the path between BS and $\mathrm{m}$. The wave-packet $|a\rangle$ reflected by BS, travels freely for a much longer time than needed to $|b\rangle$ to meet the detector $\mathrm{S}$.

parallel to the path of $|b\rangle$. Then, $|b\rangle$ is reflected by a fixed mirror $\mathrm{m}$ and flies toward a detector S.

The length of the path from the nonlinear crystal which produces the pair (not shown in the figure) to the detector $\mathrm{Q}$, is equal to that to the mirror $\mathrm{m}$. Upon the click of $\mathrm{Q}$, the mirror $\mathrm{M}$ is rotated counter-clockwise by $90^{\circ}$. Thus, if $|b\rangle$ is retro-flected inside the detector toward the input window, it exits, returns to the mirror $\mathrm{m}$, where it is reflected toward the mirror $\mathrm{M}$ which now is perpendicular to the path of $|b\rangle$. So, $|b\rangle$ is reflected back and returns to the detector S.

As far as is known to me, the experiment proposed here was not performed. However, there are other experiments, which showed that the repetition of a test of an observable on the same quantum system produced the same result as the first test, [29]. The QM itself predicts this.

So, if $|b\rangle$ is indeed retro-flected and returns to the detector, neither this time would the detector click. Under the hypothesis that the silence of the detector is due to retro-flection of $|b\rangle$ by the detector, the round trip would continue infinitely, and the detector would all the time remain silent.

However, this is impossible. An ideal, absorbing detector cannot be traversed endlessly, without the wave-packet interacting even with one particle inside. Similarly, a wave-packet cannot wander endlessly inside an ideal, absorbing detector, and never interact with a particle. In fact, it can't happen even once. Therefore, the wave-packet should have been destroyed.

Thus, the theorem is proved, ruling out the option (2). 
These two theorems leave a strange image: a wave-packet that enters a detector and does not produce a click, does not disturb even one particle in the detector. It does not remain endlessly in the detector, neither escapes from it.

The theorem below brings an additional argument against the option that the wave-packet didn't trigger a detector by exiting it.

\section{Theorem 3:}

It's impossible that a wave-packet which met an ideal detector and didn't trigger it, exited the detector unchanged.

\section{Proof:}

The proof will be done by disproving the opposite, that the wave-packet exited the detector unchanged.

The experimental arrangement bears some similarity to the Elitzur-Vaidman interaction-free measurement [30], Figure 5. From a down-conversion pair, the signal photon lands on the input beam-splitter, BS, of a Mach-Zehnder interferometer. The idler photon is sent to a detector $\mathrm{Q}$ for heralding the presence of the signal photon. The signal photon exits BS in the state

$$
|\phi\rangle=(|a\rangle+\mathrm{i}|b\rangle) / \sqrt{2} .
$$

After reflected by the mirrors $\mathrm{m},|a\rangle$ and $|b\rangle$ cross one another. In the crossing region is placed a recording plate RP (e.g. a photographic plate). The internal arms of the interferometer are of equal length. Therefore, in absence of the detector $\mathrm{A},|a\rangle$ and $|b\rangle$ produce on the RP an interference pattern. In each trial of the experiment a spot is left on the plate.

If the detector $\mathrm{A}$ is in place, the situations changes: in the trials in which both detectors $\mathrm{Q}$ and A click, the RP is not impressed, it is impressed only in the trials in which $\mathrm{Q}$ clicks, but A doesn't. That means, the number of the spots on the RP is half the number obtained in the absence of A.

Now, let's see what would be the implications if $|b\rangle$ exited the detector, unchanged.

For the trials in which Q clicks, but A remains silent, the theorem 1 says that

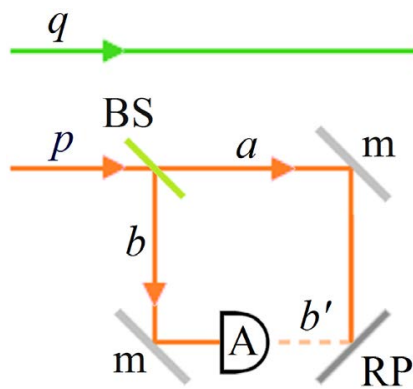

Figure 5. An experiment of Elitzur-Vaidman type. The colors are only for eye-guiding. From an idler-signal pair of down-conversion photons the idler (green) is sent to a detector $\mathrm{Q}$, and the signal (orange) to a $50 \%-50 \%$ beam-splitter, BS. $\mathrm{m}$ are fixed mirrors, A, and $\mathrm{Q}$, are ideal, absorbing detectors and $\mathrm{RP}$ is a recording (e.g. photographic) plate. The significance of the dashed path $b^{\prime}-$ pale orange-is explained in the text. 
no particle in A is perturbed. If we select only these trials, the wave-function describing them would be

$$
\begin{aligned}
\left|\Phi^{\prime}\right\rangle & =\mathrm{i}\left(|a\rangle\left|A_{1}^{\mathrm{u}}\right\rangle\left|A_{2}^{\mathrm{u}}\right\rangle \cdots+\mathrm{i}\left|b^{\prime}\right\rangle\left|A_{1}^{\mathrm{u}}\right\rangle\left|A_{2}^{\mathrm{u}}\right\rangle \cdots\right) / \sqrt{2} \\
& =\mathrm{i}\left|A_{1}^{\mathrm{u}}\right\rangle\left|A_{2}^{\mathrm{u}}\right\rangle \cdots\left(|a\rangle+\mathrm{i}\left|b^{\prime}\right\rangle\right) / \sqrt{2}
\end{aligned}
$$

Let's ignore the unperturbed particles of $\mathrm{A}$ and concentrate on the signal photon. It would be described by

$$
\left|\phi^{\prime}\right\rangle=\left(|a\rangle+\mathrm{i}\left|b^{\prime}\right\rangle\right) / \sqrt{2} .
$$

which would produce on the RP an interference pattern.

Even if $\left|b^{\prime}\right\rangle$ would differ from $|b\rangle$ by a change in phase, $\left|b^{\prime}\right\rangle=\mathrm{e}^{\mathrm{i} \phi}|b\rangle$, still an interference pattern would be produced on the RP.

However, the QM doesn't predict such effects.

To summarize these three theorems, a wave-packet which does not impress a detector, perturbs no atom in the detector, and again doesn't perturb even if it is repeatedly returned to the detector. Neither does it remain in the detector endlessly.

The only option that remains is that the wave-packet is destroyed in the detector.

\subsection{Measurements in Which the Detector Clicks- A Non-Decidable Problem}

So far we dealt with the fate of wave-packets which don't impress a detector. However, there exists also the complementary problem, if a wave-packet yes triggers a detector, what happens with the other wave-packets of the wave-function? The experiment shows that when we place detectors on them, the detectors remain silent. Therefore, according to the conclusion of the previous subsection, these wave-packets disappear. The question is, when exactly do they disappear?

Assume a dynamic experiment, i.e. the detectors are not permanently present in the setup, we introduce them when we wish. So, let's place a detector on the path of one wave-packet and concentrate on a trial in which the detector clicks. After the click we wait for a while, and place detectors on the other wave-packets. The QM predicts that the other detectors won't click, therefore their wave-packets disappeared. But when did that happen? Immediately with the click in the first detector, or when they met their own detectors?

Theorem 4: If a wave-packet of an OPWF triggers a detector, no experiment can confirm that the other wave-packets still exist.

Proof:

Two types of tests can be done on a wave-packet: a which-way test, or, interference with another wave-packet. According to the which-way tests, if one of the wave-packets made click the detector on its path, the other detectors remain silent. It is impossible to say whether the silence is because nothing impinges on 
these detectors, or, the wave-packets impinged on the detectors but were destroyed in the detectors.

Then, it remains to try if interference experiments can provide more information.

Figure 6 illustrates a Sciarrino-type experiment [31] [32]. On two 50\% $50 \%$ beam-splitters, $\mathrm{BS}_{\mathrm{A}}$ and $\mathrm{BS}_{\mathrm{B}}$, land identical photons from a degenerate-down-conversion pair. From each photon, the reflected part $|a\rangle(|b\rangle)$ is sent to another $50 \%-50 \%$ beam-splitter, BS, while the transmitted part $\left|a^{\prime}\right\rangle$ $\left(\left|b^{\prime}\right\rangle\right)$ is sent to a $2 \mathrm{D}$ detector array, DA, consisting in miniature absorbing detectors. ${ }^{7}$ The total wave-function of the photons at this step, is

$$
|\Phi\rangle=\frac{1}{2}\left(\mathrm{i}|a\rangle+\left|a^{\prime}\right\rangle\right)\left(\mathrm{i}|b\rangle+\left|b^{\prime}\right\rangle\right)=\frac{1}{\sqrt{2}}\left(\mathrm{i} \frac{|a\rangle\left|b^{\prime}\right\rangle+|b\rangle\left|a^{\prime}\right\rangle}{\sqrt{2}}-\frac{|a\rangle|b\rangle-\left|a^{\prime}\right\rangle\left|b^{\prime}\right\rangle}{\sqrt{2}}\right) \text {. }
$$

In continuation, we will not be interested in two detections on the same side of the setup, i.e. both beyond BS or both on the DA. So, we will work only with the state

$$
|\psi\rangle=\left(|a\rangle\left|b^{\prime}\right\rangle+|b\rangle\left|a^{\prime}\right\rangle\right) / \sqrt{2} .
$$

If the detector $\mathrm{A}$ is not present in the setup, the wave-packets $|a\rangle$ and $|b\rangle$ land on the beam-splitter BS, where they undergo the transformation

$$
|a\rangle \rightarrow(\mathrm{i}|c\rangle+|d\rangle) / \sqrt{2}, \quad|b\rangle \rightarrow(|c\rangle+\mathrm{i}|d\rangle) / \sqrt{2} .
$$

Introducing this in (9) there results

$$
|\psi\rangle \rightarrow \frac{1}{\sqrt{2}}\left(|c\rangle \frac{\left|a^{\prime}\right\rangle+\mathrm{i}\left|b^{\prime}\right\rangle}{\sqrt{2}}+\mathrm{i}|d\rangle \frac{\left|a^{\prime}\right\rangle-\mathrm{i}\left|b^{\prime}\right\rangle}{\sqrt{2}}\right) .
$$

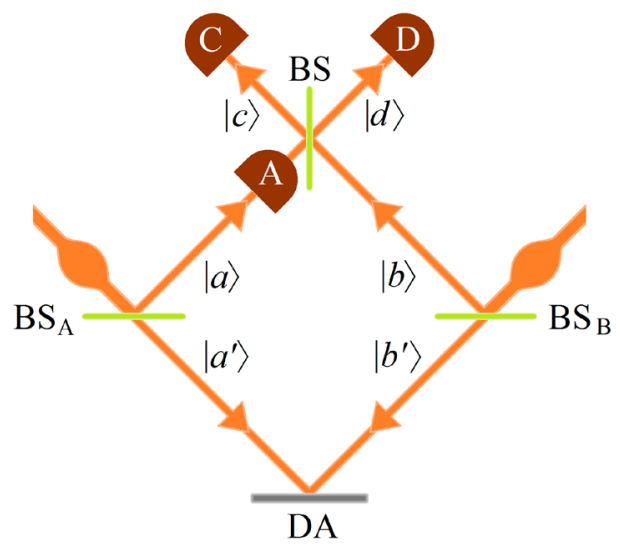

Figure 6. A Sciarrino-type experiment. The colors are only for eye-guiding. $\mathrm{BS}_{\mathrm{A}}, \mathrm{BS}_{\mathrm{B}}$ and BS, are $50 \%-50 \%$ beam-splitters. They are illuminated by identical photons from a degenerate-down-conversion pair. A, C, D, are ideal, absorbing detectors, $\mathrm{C}$ and $\mathrm{D}$ are fixed, and $\mathrm{A}$ is removable. $\mathrm{DA}$ is a recording $2 \mathrm{D}$ array of miniature, absorbing detectors. They record the time and the $x-y$ coordinates of the absorbed photon in each trial and trial of the experiment. The time is also recorded for each detection in C, and for each detection in $\mathrm{D}$.

${ }^{7}$ Such a net of detectors was used in the experiments described in [33] [34]. 
This state tells that if one collects separately the recordings in all the trials in which the detector $\mathrm{C}$ clicked, the detector array would have recorded an interference pattern. Analogously, if one collects separately the recordings in all the trials in which the detector D clicked, another interference pattern is obtained.

However, if the detector A is introduced, there won't be interference patterns.

The case of interest for the theorem 4 is when A clicks. As A is an ideal, absorbing detector, this click testifies that the wave-packet $|a\rangle$ was absorbed, so what exits A is vacuum. At this step Equation (9) becomes,

$$
|\psi\rangle=\left(|0\rangle\left|b^{\prime}\right\rangle+|b\rangle\left|a^{\prime}\right\rangle\right) / \sqrt{2} \text {. }
$$

Introducing the transformation (10) (the RHS part of the transformation) there results

$$
|\psi\rangle \rightarrow \frac{1}{\sqrt{2}}\left(|0\rangle\left|b^{\prime}\right\rangle+\frac{|c\rangle+\mathrm{i}|d\rangle}{\sqrt{2}}\left|a^{\prime}\right\rangle\right) .
$$

Then, if no click is recorded in the detector $\mathrm{C}$ or $\mathrm{D},(13)$ becomes

$$
|\vartheta\rangle=\left(|0\rangle\left|b^{\prime}\right\rangle+|0\rangle\left|a^{\prime}\right\rangle\right) / \sqrt{2}=|0\rangle\left(\left|b^{\prime}\right\rangle+\left|a^{\prime}\right\rangle\right) / \sqrt{2} \text {. }
$$

It seems that the wave-packets $\left|a^{\prime}\right\rangle$ and $\left|b^{\prime}\right\rangle$ would produce interference, confirming that although the wave-packet $|a\rangle$ was detected, $\left|a^{\prime}\right\rangle$ survived.

However, all the wave-functions (12) thru (14) are wrong. They ignore the involvement of the particles in the detector A. As long as the quantum description can still be used, (14) should be replaced by

$$
\left.|\Phi\rangle=|0\rangle\left(\left|b^{\prime}\right\rangle\left|A_{1}^{\mathrm{p}}\right\rangle\left|A_{2}^{\mathrm{p}}\right\rangle \cdots+\left|a^{\prime}\right\rangle\left|A_{1}^{\mathrm{u}}\right\rangle\right\rangle\left|A_{2}^{\mathrm{u}}\right\rangle \cdots\right) / \sqrt{2} .
$$

So, no interference is possible.

That does not prove that at the click of A, the wave-packet $\left|a^{\prime}\right\rangle$ is destroyed. It only proves that attempting to check by an interference experiment whether $\left|a^{\prime}\right\rangle$ exists after $|a\rangle$ is detected, is doomed to fail.

Thus, the theorem 4 is proved.

To summarize: if $|a\rangle$ was detected, then, when $\left|a^{\prime}\right\rangle$ touches the detectors DA it produces no detection.

There are two possible explanations of that. 1) $\left|a^{\prime}\right\rangle$ disappeared in consequence of the detection of $|a\rangle$, or, 2) it continued to exist and was destroyed in the detectors DA.

We wanted to know which explanations is the correct one. It turned up to be non-decidable. Neither which-way experiments, nor interference experiments, prove whether the wave-packet $\left|a^{\prime}\right\rangle$ continues to exist or is destroyed if $|a\rangle$ is detected.

\section{Stochastic Evolution of a Microscopic System}

In the Section 2.1 of [10], GPR developed an equation for a stochastically evolving system described by a state-function $|\psi\rangle$. They started from a very general equation of this type, the Itô equation, 


$$
\mathrm{d}|\psi\rangle=\left(\hat{C} \mathrm{~d} t+\sum_{n} \hat{A}_{n} \mathrm{~d} B_{n}\right)|\psi\rangle .
$$

In this equation $\hat{C}$ and $\left\{\hat{A}_{n}\right\}$ are operators, and $\left\{B_{n}\right\}$ is a set of real, Wiener processes. ${ }^{8}$ Since in this text we are going to work with a single operator $\hat{A}$ we rewrite (16) for our case,

$$
\mathrm{d}|\psi\rangle=(\hat{C} \mathrm{~d} t+\hat{A} \mathrm{~d} B)|\psi\rangle .
$$

A Wiener process $B$ is stochastic, the quantity $B$ may take at a time $t$ from the beginning of the trial, an arbitrary value, on condition that the differential element $\mathrm{d} B(t)=B(t+\mathrm{d} t)-B(t)$ obeys certain constraints. For a white noise these constraints are:

$$
\overline{\mathrm{d} B(t)}=0, \text { i) } \overline{[\mathrm{d} B(t)]^{2}}=\gamma \mathrm{d} t, \text { ii) }
$$

where the average is taken over all the trails, at the time $t$ measured since the beginning of each trial. $\gamma$ is a parameter expressing the intensity of the noise.

Replacing the non-Hermitian part of the operator $\hat{C}$ with $-\mathrm{i} \hat{H}$, where $\hat{H}$ is the Hamiltonian, Equations (16) and (17) would look like a Schrödinger equation, though with additional terms besides the Hamiltonian. The presence of additional terms yields a different solution, and non-normalized, in comparison with the solution of the standard Schrödinger equation. For normalizing

$$
|\phi\rangle_{t}=|\psi\rangle_{t} /\left[{ }_{t}\langle\psi \mid \psi\rangle_{t}\right]^{1 / 2}
$$

GPR did the appropriate changes in (16). Next, applying the Itô formalism [35], they obtained a non-linear SSE, which for a single Hermitian operator $\hat{A}$, reads

$$
\left.\left.\mathrm{d}|\phi\rangle_{t}=\left\{-\mathrm{i} \hat{H} \mathrm{~d} t-\frac{1}{2} \gamma[\hat{A}-R(t)]^{2} \mathrm{~d} t+[\hat{A}-R(t)] \mathrm{d} B(t)\right\}|\phi\rangle_{t}, \mathrm{i}\right) \quad R(t)={ }_{t}\langle\phi|\hat{A}| \phi\rangle_{t} \cdot \mathrm{ii}\right)^{9}
$$

Remark 2: For Equation (20) to reduce to the Schrödinger equation, the last two terms in (20i) must bring a negligible contribution to the solution, in comparison with $\mathrm{i} \hat{H} \mathrm{~d} t$. It will be seen in the end of this section and in the next section that as long as a system consists in a few microscopic components the last two terms have indeed a negligible effect.

Remark 3: It was proved in [13] that a nonlinear equation would allow faster than light communication. However, the stochastic character of the noise impedes such a possibility.

Further, GPR tried to prove-Section 2.2. of [10] — that a system evolving by the non-linear SSE obtained by them ends up in an eigenfunction of the set

${ }^{8} \mathrm{~A}$ Wiener process refers to a parameter $B(t)$ which varies continuously in time, however, the value of the increment $\mathrm{d} B(t)$ jumps from time to time, so that the derivative of $B(t)$ has points of discontinuity.

${ }^{9}$ Detailed explanations of these calculi are given in the section 7 of [14]. 
$\left\{\hat{A}_{n}\right\}$. By analogy, our Equation (20) with a single operator, $\hat{A}$, should have as solution one of the eigenstates of $\hat{A}$. The purpose of this section and of the next one is to check in detail this inference.

GPR decided to ignore in the influence of $\hat{H}$ during the process of localization, motivating:

"Since we are interested here in discussing the physical effects of the new terms, we disregard for the moment the Schrödinger part of the dynamical equation."

This decision is not trivial. The effect of $\hat{H}$ may indeed be ignored if the localization occurs extremely quickly on the time scale of the evolution by $\hat{H}$. However, if the Hamiltonian contains an interaction term which represents the cause of the locolization of the system, the localization occurs at the pace of the evolution by $\hat{H}$. This will be the case in this text. Fortunately, it will be shown that $\hat{H}$ cancels out in the calculi, simplifying them.

For understanding the physical ground of the calculi in continuation the following explanation is necessary:

Remark 4: consider that the wave-function $|\phi\rangle$ is of the form

$$
|\phi\rangle=\sum_{j} \boldsymbol{a}_{j}(t)\left|a_{j}\right\rangle,
$$

where $\left|a_{j}\right\rangle$ is the eigenstate corresponding to the eigenvalue $a_{j}$. The experiment shows that the probability of obtaining the result $a_{j}$, is $\left|\boldsymbol{a}_{j}(t)\right|^{2}$. However, from phenomenological point of view, $\left\{\left|\boldsymbol{a}_{j}(t)\right|^{2}\right\}$ are the intensities of the eigenwaves. The connection between the intensity of the eigenwave $\left|a_{j}\right\rangle$ and the probability to obtain, in a trial of the experiment, the result $a_{j}$, is simple: the greater is the intensity of an eigenwave, the greater is the probability that it would be detected. So, when we say that in a certain trial of an experiment occurs localization, say, on $\left|a_{j}\right\rangle$, the meaning is that in that trial the intensity $\left|\boldsymbol{a}_{j}(t)\right|^{2}$ of the eigenwave $\left|a_{j}\right\rangle$ increases to 1 , while the intensities of all the other eigenwaves decrease to zero.

For proving that the CSL model predicts localization, GPR followed a procedure that is exposed below with a single operator $\hat{A}$, and without ignoring the Hamiltonian.

Let $\hat{P}_{j}$ be the projection operator on the eigenstate $\left|a_{j}\right\rangle \cdot|\phi\rangle$ can be expanded as follows:

$$
|\phi\rangle=\sum_{j} \hat{P}_{j}|\phi\rangle .
$$

From the above relation one immediately obtains

$$
\left.\hat{A}|\phi\rangle=\sum_{j} a_{j} \hat{P}_{j}|\phi\rangle, \text { i }\right) \quad \hat{A}^{2}|\phi\rangle=\sum_{j} a_{j}^{2} \hat{P}_{j}|\phi\rangle \text {, ii) } R(t)=\sum_{j}\left\langle\phi\left|a_{j} \hat{P}_{j}\right| \phi\right\rangle=\sum_{j} a_{j}\left\langle\phi\left|\hat{P}_{j}\right| \phi\right\rangle \text {. iii) }
$$

Obviously from (21) and (22), 


$$
\hat{P}_{j}|\phi\rangle=\boldsymbol{a}_{j}(t)\left|a_{j}\right\rangle \text {, i) }\left\langle\phi\left|\hat{P}_{j}\right| \phi\right\rangle=\left|\boldsymbol{a}_{j}(t)\right|^{2} \text {. ii) }
$$

It is more useful for our purpose to work with the intensities, than with the amplitudes. Introducing (22) in (20), using equalities (23), and projecting on the eigenstate $\left|a_{m}\right\rangle$,

$$
\mathrm{d} \hat{P}_{m}|\phi\rangle=\left\{-\mathrm{i} E_{m} \mathrm{~d} t-\frac{1}{2} \gamma\left[a_{m}-R(t)\right]^{2} \mathrm{~d} t+\left[a_{m}-R(t)\right] \mathrm{d} B(t)\right\} \hat{P}_{m}|\phi\rangle .
$$

The following identity results from the rules of the Itô stochastic calculus-see also section 7.3 in [14],

$$
\mathrm{d}\left\langle\phi\left|\hat{P}_{m}\right| \phi\right\rangle=\left[\mathrm{d}\langle\phi| \hat{P}_{m}\right] \hat{P}_{m}|\phi\rangle+\langle\phi| \hat{P}_{m}\left[\mathrm{~d} \hat{P}_{m}|\phi\rangle\right]+\left[\mathrm{d}\langle\phi| \hat{P}_{m}\right]\left[\mathrm{d} \hat{P}_{m}|\phi\rangle\right] .
$$

We will calculate the components of this equation using (21)-(25) and the properties (18) of $\mathrm{d} B$.

$$
\begin{aligned}
& \mathrm{d}\left\langle\phi\left|\hat{P}_{m}\right| \phi\right\rangle=\mathrm{d}\left[\left|\boldsymbol{a}_{m}(t)\right|^{2}\right], \\
& {\left[\mathrm{d}\langle\phi| \hat{P}_{m}\right] \hat{P}_{m}|\phi\rangle+\langle\phi| \hat{P}_{m}\left[\mathrm{~d} \hat{P}_{m}|\phi\rangle\right]} \\
& =\left\{-\gamma\left[a_{m}-R(t)\right]^{2} \mathrm{~d} t+2\left[a_{m}-R(t)\right] \mathrm{d} B(t)\right\}\left|\boldsymbol{a}_{m}(t)\right|^{2}, \\
& {\left[\mathrm{~d}\langle\phi| \hat{P}_{m}\right]\left[\mathrm{d} \hat{P}_{m}|\phi\rangle\right] \approx\left[a_{m}-R(t)\right]^{2}\left|\boldsymbol{a}_{m}(t)\right|^{2} \gamma \mathrm{d} t .}
\end{aligned}
$$

In the calculus of $\left[\mathrm{d}\langle\phi| \hat{P}_{m}\right]\left[\mathrm{d} \hat{P}_{m}|\phi\rangle\right]$ we ignored terms with $(\mathrm{d} t)^{2}$ and the terms with $\mathrm{d} B \mathrm{~d} t$, vis-à-vis the terms with $\mathrm{d} B$ and the terms with $\mathrm{d} t$, since $\mathrm{d} t$ is usually extremely small.

Let's notice that the terms with the Hamiltonian cancelled one another out.

Introducing the RHSs of (27) in (26) there results

$$
\mathrm{d}\left[\left|\boldsymbol{a}_{m}(t)\right|^{2}\right]=2\left|\boldsymbol{a}_{m}(t)\right|^{2}\left[a_{m}-R(t)\right] \mathrm{d} B(t) .
$$

One may be eluded by the form of this equation and think that dividing both sides by $\left|\boldsymbol{a}_{m}(t)\right|^{2}$, it is possible to integrate and get,

$$
\left|\boldsymbol{a}_{m}(t)\right|^{2}=\left|\boldsymbol{a}_{m}\left(t_{0}\right)\right|^{2} \exp \left\{2 \int_{t_{0}}^{t}\left[a_{m}-R(\tau)\right] \mathrm{d} B(\tau)\right\} .
$$

However, (23iii) and (24ii) show that $R(t)$ also depends on the set $\left\{\left|\boldsymbol{a}_{j}(t)\right|^{2}\right\}$. Then, the solution of (28) should rather be calculated iteratively, each iteration corresponding to an elementary interval $\mathrm{d} t$. One can start from $t_{0}$, a time before the noise began to act on the quantum system, so all $\left\{\left|\boldsymbol{a}_{j}\left(t_{0}\right)\right|^{2}\right\}$ are known from the initial wave-function. A given iteration would calculate the set of the differentials $\left\{\mathrm{d}\left[\left|\boldsymbol{a}_{j}(t)\right|^{2}\right]\right\}$ using (28), and the next iteration will update $\left\{\left|\boldsymbol{a}_{j}\left(t_{0}\right)\right|^{2}\right\}$ and calculate a new set $\left\{\mathrm{d}\left[\left|\boldsymbol{a}_{j}(t)\right|^{2}\right]\right\}$. The new differentials will be used in the iteration that follows, and so on. A specific example is given in Subsection 5.2.

Though, (29) is useful for getting an approximate evaluation of the evolution of $\left\{\left|\boldsymbol{a}_{j}(t)\right|^{2}\right\}$ during a detection. Let's check for instance, under which conditions the intensity $\left|\boldsymbol{a}_{m}(t)\right|^{2}$ may vanish. For such an end, the value of the ex- 
ponential in the RHS of (29) should be $-\infty$.

In practice, if the exponent in the RHS of (29) accumulates at a time $t_{1}$ a value, say, -13 , it would render $\left|\boldsymbol{a}_{m}\left(t_{1}\right)\right|^{2}$ quite small, of the order of $\left|\boldsymbol{a}_{m}\left(t_{0}\right)\right|^{2} \times 2.3 \times 10^{-6}$.

Admitting that the eigenvalues $\left\{a_{j}\right\}$ are dimensionless and of the order of unity, e.g. the spin projection of a spin 1 boson with $\hbar$ being set equal to $1, R$ would be of the same order of magnitude.

According to (18ii), $|\mathrm{d} B|$ fluctuates around $\sqrt{\gamma \mathrm{d} t}$. In the section 5 we will give a numerical example which imposes $\mathrm{d} t$ to be about $5 \times 10^{-13} \mathrm{~s}$, and $\gamma=800 \mathrm{~s}^{-1}$ which is by very many orders of magnitude greater than $\gamma$ values provided by experiments on the CSL model [36].

So, a simple choice for $\mathrm{d} B$ would be $\pm 2 \times 10^{-5}$.

According to (18i), from $t_{0}$ to $t_{1}$ the sign of $\mathrm{d} B$ may change an arbitrary number of times, even after each $\mathrm{d} t$, despite of that, let's admit that $\mathrm{d} B$ is orchestrated in some way to be always of sign opposite to $\left[a_{m}-R(\tau)\right]$.

With these data, for the exponent in the RHS of (29) to accumulate until the time $t_{1}$ the value -13 , the integral in (29) has to be the sum of $6.5 /\left(2 \times 10^{-5}\right)=3.25 \times 10^{5}$ pieces. As each such piece lasts $5 \times 10^{-13} \mathrm{~s}$, the decrease of $\left|\boldsymbol{a}_{m}\right|^{2}$ would take $3.25 \times 10^{5} \times 5 \times 10^{-13} \mathrm{~s}=162.5 \mathrm{~ns}$. This is a long time. For comparison, as we will see in Subsection 5.3, in a proportional counter the interval of time until the output signal begins to rise is $0.1 \mathrm{~ns}$, an interval cca. 1.6 $\times 10^{3}$ times shorter. And that, besides the fact that the constraint (18i) does not allow $\mathrm{d} B$ to be always of sign opposite to $\left[a_{m}-R\right]$.

The conclusion is not that the CSL model is wrong, to the contrary. Since the calculus above was done for a simple system, e.g. one quantum particle, it was expected according to the very principle of the model that no localization can occur. As the model says, the localization appears in a multi-component system. A totally different situation will be described in the next section.

\section{The CSL Model and the Detection Process}

In this section the CSL model is going to be tested on the process occurring inside a detector when a quantum particle enters and more and more particles from the detector are perturbed. As said in Section 2, we do our rationale on a detector working in the proportional regime. It will be supposed that the initial wave-function is a OPWF of the form

$$
\left.|\Psi\rangle_{t_{0}}=\boldsymbol{a}_{1}\left(t_{0}\right)\left|\Psi_{1}\right\rangle+\boldsymbol{a}_{2}\left(t_{0}\right)\left|\Psi_{2}\right\rangle+\boldsymbol{a}_{3}\left(t_{0}\right)\left|\Psi_{3}\right\rangle, \text { i) } \sum_{k=1}^{3}\left|\boldsymbol{a}_{k}(t)\right|^{2}=1, \text { ii }\right)
$$

where $\left|\Psi_{k}\right\rangle$ indicates the presence of a quantum particle on the path $k$. It will also be supposed that an ideal detector is placed on the wave-packet $\left|\Psi_{1}\right\rangle$; no detectors will be placed on the other wave-packets. It is expected that the model make the following predictions:

1) If the detector clicks, the intensity of the wave-packet $\left|\Psi_{1}\right\rangle$ has increased to 1 . The number of electron-ion pairs in the detector becomes equal to the 
number of pairs that would have been produced if the initial wave-function possessed only one wave-packet, $\left|\Psi_{1}\right\rangle$, (so that the detection by the ideal detector would have been sure).

2) If the detector remains silent, $\left|\boldsymbol{a}_{1}(t)\right|^{2}$ decreases to zero according to the theorem 1 which requires that the number of electron-ion pairs in the detector, become null.

\subsection{The Test of a Many-Component System-The Process inside a Detector}

With the impingement of $\left|\Psi_{1}\right\rangle$ on the detector, the OPWF changes into an entanglement. As described in Section 2, the visitor wave-packet may cause a couple of primary ionizations, and then, each primary electron is dragged by the electric field to the multiplication region. Thus, the entanglement takes the form

$$
\left.\left.|\Phi\rangle_{t}=\boldsymbol{a}_{1}(t) \sum_{j=1}^{\infty} c_{j}(t)|j\rangle+\boldsymbol{a}_{2}(t)\left|\Psi_{2}\right\rangle|0\rangle+\boldsymbol{a}_{3}(t)\left|\Psi_{3}\right\rangle|0\rangle, \mathrm{i}\right) \sum_{j=0}^{\infty}\left|c_{j}(t)\right|^{2}=1, \mathrm{ii}\right)
$$

where $j$ is the number of electron-ions pairs in the detector. The upper limit of $j$ is the number of atoms in the detector, a number known only as an order of magnitude. But this number is huge in comparison with the number of electron-ion pairs that may appear, for which reason it was set to $\infty$.

GPR developed an equation for the evolution of a set of identical particles section 3.1 in [10],

$$
\mathrm{d}|\psi\rangle=\left\{-\mathrm{i} \hat{H} \mathrm{~d} t-\frac{1}{2} \gamma \int \mathrm{d} \mathbf{r} \hat{N}^{2}(\mathbf{r}) \mathrm{d} t+\int \mathrm{d} \mathbf{r} \hat{N}(\mathbf{r}) \mathrm{d} B(\mathbf{r}, t)\right\}|\psi\rangle,
$$

where the operator $\hat{N}(\mathbf{r})$ was attached to the density of number of particles at the position $\mathbf{r}$, and $\langle\hat{N}(\mathbf{r})\rangle_{t}$ is the average number of particles at the position $\mathbf{r}$ and time $t$. Details on how $\hat{N}(\mathbf{r})$ can look like, on its eigenfunctions and eigenvalues, can be found in [14] Section 8.1 and [10] Section 3.1.

Normalizing the wave-function (32) as we did in the former section - relation (19) - one gets

$$
\begin{aligned}
\mathrm{d}|\phi\rangle_{t}= & \left\{-\mathrm{i} \hat{H} \mathrm{~d} t-\frac{1}{2} \gamma \int \mathrm{d} \mathbf{r}\left[\hat{N}(\mathbf{r})-\langle\hat{N}(\mathbf{r})\rangle_{t}\right]^{2} \mathrm{~d} t\right. \\
& \left.+\int \mathrm{d} \mathbf{r}\left[\hat{N}(\mathbf{r})-\langle\hat{N}(\mathbf{r})\rangle_{t}\right] \mathrm{d} B(\mathbf{r}, t)\right\}|\phi\rangle_{t}
\end{aligned}
$$

GPR obtained Equation (32) for a fixed number of particles. However, in a detector which could click the situation is different; the visitor particle perturbs more and more particles from the detector as the time passes. In the case of a proportional counter, inside the avalanche region the number of electron-ion pairs increases exponentially in time as explained in Section 2. That would require that the Hamiltonian comprise creation and annihilation operators, e.g.

$$
\hat{G}=\hat{a}_{\mathrm{e}}^{\dagger}(\mathbf{r}, t) \hat{a}_{\text {ion }}^{\dagger}(\mathbf{r}, t) \hat{a}_{\text {atom }}(\mathbf{r}, t)+\text { c.c. },
$$


and the increase in time of the number of PPCs would make $\hat{H}$ more and more complicated. Fortunately, in our calculi the Hamiltonian will be eliminated, as it was eliminated in the calculi in Section 4.

Another problem is that in part of the collisions are not produced electron-ion pairs but excited atoms on high levels. Then, the atoms de-excite through emission of high energy photons, which set free new electrons by the photo-electric effect. These electrons ionize atoms, starting secondary avalanches, i.e. avalanches which do not originate in primary electrons.

For avoiding these complications we shall make a couple of assumptions, part of them adopted also in [26] chapter 6, section III A.

*) Since the probability of secondary avalanches in the proportional counter is small, we assume that no free electron is lost, each free electron undergoes a PPC.

"The only multiplication process is through electron collisions (any photoelectric effects are neglected), that no electrons are lost to negative ion formation, and that space-charge effects are negligible”. [26]

**) In the present study there is no benefit from assuming that $\mathrm{d} B$ is position dependent inside the detector. This fact will appear more convincing in Subsection 5.2. Therefore, inside the detector, $\mathrm{d} B$ will be considered as varying only in time.

The space-distribution of the electrons and ions inside the detector is also going to be irrelevant in our analysis. Given also the assumption $\left({ }^{\star *}\right)$, we can work with a simpler equation

$\left.\left.\mathrm{d}|\Phi\rangle_{t}=\left\{-\mathrm{i} \hat{H} \mathrm{~d} t-\frac{1}{2} \gamma\left(\hat{N}-\langle\hat{N}\rangle_{t}\right)^{2} \mathrm{~d} t+\left(\hat{N}-\langle\hat{N}\rangle_{t}\right) \mathrm{d} B(t)\right\}|\Phi\rangle_{t}, \mathrm{i}\right)\langle\hat{N}\rangle_{t}={ }_{t}\langle\Phi|\hat{N}| \Phi\rangle_{t} . \mathrm{ii}\right)$

where the operator $\hat{N}$ is associated with the total number of electron-ion pairs in the detector, $\hat{N}|j\rangle=j|j\rangle$. Applying it to the wave-function (31i) and calculating $\langle\hat{N}\rangle_{t}$ according to (35ii)

$$
\left.\left.\langle\hat{N}\rangle_{t}=\left|\boldsymbol{a}_{1}(t)\right|^{2} Y(t), \mathrm{i}\right) \quad Y(t)=\sum_{j=1}^{\infty} j\left|c_{j}(t)\right|^{2} . \mathrm{ii}\right)
$$

Remark 5: the formulas (36) may eventually confuse the reader, so, it is better to stress the difference between $\langle\hat{N}\rangle_{t}$ and $Y(t)$. As one can infer from (36i), $Y(t)$ is number of electron-ion pairs that would be present in the detector if $\left|\boldsymbol{a}_{1}(t)\right|^{2}$ were equal to 1 , i.e. if the wave-function consisted in only one wave-packet, $\left|\Psi_{1}\right\rangle$. In this case the ideal detector would have reported the incident particle, with certainty. However, the wave-function contains three wave-packets, not one. Inspecting the wave-function (31) one can infer that the $Y(t)$ pairs could be found in the detector, with the probability $\left|\boldsymbol{a}_{1}(t)\right|^{2}$, and with a probability $1-\left|\boldsymbol{a}_{1}(t)\right|^{2}$ no pairs would be found. $\langle\hat{N}\rangle_{t}$ takes in consideration 
this situation.

In continuation, for finding out whether the model can predict (a) or (b), we try to get an equation for the evolution in time of $\left|\boldsymbol{a}_{1}\right|^{2}$. We begin by projecting Equation (35i) onto the Fock state of $m$ pairs,

$$
\begin{aligned}
& \mathrm{d} \hat{P}_{m}|\Phi\rangle_{t}=\hat{P}_{m} \mathrm{~d}|\Phi\rangle_{t} \\
& =-\left\{\mathrm{i} E_{m} \mathrm{~d} t+\frac{1}{2} \gamma\left[\langle\hat{N}\rangle_{t}-m\right]^{2} \mathrm{~d} t+\left[\langle\hat{N}\rangle_{t}-m\right] \mathrm{d} B(t)\right\} \hat{P}_{m}|\Phi\rangle_{t}
\end{aligned}
$$

and use that in the identity (26). The result is similar with (28) with the only difference that-see (31) - instead of (24) one has

$$
\left.\left.\hat{P}_{m}|\Phi\rangle_{t}=\boldsymbol{a}_{1}(t) c_{m}(t)|m\rangle, \mathrm{i}\right)\left\langle\Phi\left|\hat{P}_{m}\right| \Phi\right\rangle_{t}=\left|\boldsymbol{a}_{1}(t)\right|^{2}\left|c_{m}(t)\right|^{2} . \mathrm{ii}\right)
$$

Doing this replacement in (28),

$$
\left[\mathrm{d}\left|\boldsymbol{a}_{1}(t)\right|^{2}\right]\left|c_{m}(t)\right|^{2}+\left|\boldsymbol{a}_{1}(t)\right|^{2} \mathrm{~d}\left|c_{m}(t)\right|^{2}=2\left[m-\langle\hat{N}\rangle_{t}\right]\left|c_{m}(t)\right|^{2}\left|\boldsymbol{a}_{1}(t)\right|^{2} \mathrm{~d} B(t)
$$

For getting rid of $\mathrm{d}\left|c_{m}(t)\right|^{2}$ we do summation over $m$, use (31ii) and (36ii), then, replace $\langle\hat{N}\rangle_{t}$ by $(36 \mathrm{i})$,

$$
\mathrm{d}\left|\boldsymbol{a}_{1}(t)\right|^{2}=2\left[1-\left|\boldsymbol{a}_{1}(t)\right|^{2}\right] Y(t)\left|\boldsymbol{a}_{1}(t)\right|^{2} \mathrm{~d} B(t) .
$$

\subsection{General Implications}

Comparing Equation (40) with (28), it's obvious that the presence of $Y(t)$ in the former-see Remark 5-makes $\left|\boldsymbol{a}_{1}(t)\right|^{2}$ vary in time, by much bigger steps than in the case of one single particle. According to the explanations in Section 2 and the assumption $\left.{ }^{*}\right), Y$ increases exponentially in time,

$$
Y_{k}=n_{0} 2^{k}
$$

where $n_{0}$ is the number of the primary electrons and $k$ is the number of the last PPC generation.

If the physical conditions constraint

$$
0 \leq\left|\boldsymbol{a}_{1}(t)\right|^{2} \leq 1
$$

is obeyed during the detection process, Equation (40) shows that $\left|\boldsymbol{a}_{1}\right|^{2}$ would increase if $\mathrm{d} B>0$, and decrease if $\mathrm{d} B<0$, since $Y$ is positive. For sufficiently high values of $Y, \mathrm{~d} B>0$ would augment $\left|\boldsymbol{a}_{1}\right|^{2}$ toward 1 -expectation (a), while $\mathrm{d} B<0$ would lower $\left|\boldsymbol{a}_{1}\right|^{2}$ toward 0-expectation (b).

A specific example is given in the next subsection.

It would be appealing to divide on both sides of (40) by $\left[1-\left|\boldsymbol{a}_{1}(t)\right|^{2}\right]\left|\boldsymbol{a}_{1}(t)\right|^{2}$ and integrate the equation, if $\mathrm{d} B$ were a known function. But it isn't. What one can do is to solve (40) iteratively, by assuming some sequence $\mathrm{d} B_{1}, \mathrm{~d} B_{2}, \cdots$

However, the validity of (40) is limited: if from some iteration results $\mathrm{d}\left|\boldsymbol{a}_{1}\right|^{2}<0$ with $\left.\left.|\mathrm{d}| \boldsymbol{a}_{1}\right|^{2}|>| \boldsymbol{a}_{1}\right|^{2}$, in the next iteration one will get $\left|\boldsymbol{a}_{1}\right|^{2}$ negative, which is non-physical. The following limitation emerges straightforwardly from 
$(40)$

$$
\left|2 Y(t)\left[1-\left|\boldsymbol{a}_{1}(t)\right|^{2}\right] \mathrm{d} B(t)\right|<1 .
$$

If in some iteration there results $\mathrm{d}\left|\boldsymbol{a}_{1}\right|^{2}>\left(1-\left|\boldsymbol{a}_{1}\right|^{2}\right)$, in the next iteration $\left|\boldsymbol{a}_{1}\right|^{2}$ would exceed 1, which is non-physical too. Obviously, if $\mathrm{d}\left|\boldsymbol{a}_{1}\right|^{2}>\left(1-\left|\boldsymbol{a}_{1}\right|^{2}\right)$, $\mathrm{d}\left|\boldsymbol{a}_{1}\right|^{2}$ is positive. There emerges immediately from (40)

$$
0<2 Y(t)\left|\boldsymbol{a}_{1}(t)\right|^{2} \mathrm{~d} B(t)<1 .
$$

Adding up these two inequalities side by side, one gets the limits between which Equation (40) is valid:

$$
-1<2 Y(t) \mathrm{d} B(t)<2 .
$$

\subsection{A numerical Evaluation}

We are going to check whether the CSL model predicts the results (a) and (b), by following the process in a proportional counter of cylindrical symmetry-Figure 1 -containing a mixture of $95 \% \mathrm{Xe}$ and $5 \% \mathrm{CO}_{2}$. The parameters of this counter, suitable for a number of PPC generations equal to 12, are detailed in the Appendix.

Table 1 shows the evolution of $\left|\boldsymbol{a}_{1}\right|^{2}$ along the detection process. As initial values are taken $\left|\boldsymbol{a}_{1}\left(t_{0}\right)\right|^{2}=0.4$, and $n_{0}=10 . T_{k}$ denotes the time elapsed between the $k^{\text {th }}$ and the $(k+1)^{\text {th }}$ PPCs generation. It contains a couple of elementary intervals $\mathrm{d} t$, and appears in the tables in units $\mathrm{d} t$-as said in section 4 , $\mathrm{d} t$ is chosen equal to $5 \times 10^{-13}$ s. For each $T_{k}$, we replace $Y(t)$ by the RHS of (41). It is quite a rough approximation, since not all the PPCs in a generation occur strictly simultaneously, though, what we seek out for the moment is a general image of the unfolding of the process.

Table 1. (a) (part 1). (b) (part 2).

(a)

\begin{tabular}{cccccccc}
\hline$k$ & 1 & 2 & 3 & 4 & 5 & 6 & 7 \\
\hline$T_{k}$ & 26 & 23 & 22 & 20 & 18 & 17 & 15 \\
$\Sigma^{(k)} \operatorname{sign}(\mathrm{d} B)$ & -4 & 1 & 0 & 2 & 0 & -1 & 3 \\
$Y_{k}$ & 20 & 40 & 80 & 160 & 320 & 640 & 1280 \\
$|\boldsymbol{a}|^{2}$ & 0.4 & 0.4 & 0.4 & 0.4 & 0.4 & 0.39 & 0.42 \\
\hline
\end{tabular}

(b)

\begin{tabular}{cccccc}
\hline$k$ & 8 & 9 & 10 & 11 & 12 \\
\hline$T_{k}$ & 14 & 13 & 12 & 11 & 10 \\
$\Sigma^{(k)} \operatorname{sign}(\mathrm{d} B)$ & -2 & -1 & 2 & 3 & 0 \\
$Y_{k}$ & 2560 & 5120 & 10,240 & 20,480 & 40,960 \\
$\left|\boldsymbol{a}_{1}\right|^{2}$ & 0.4 & 0.28 & 0.3 & 0.98 & 0.99999 \\
\hline
\end{tabular}


The rest of the data in the $k^{\text {th }}$ column correspond to the end of the interval $T_{k}$. The symbol $\Sigma^{(k)} \operatorname{sign}(\mathrm{d} B)$ denotes the sum of the signs of $\mathrm{d} B$ within $T_{k}$. Let's remind that in the section 4 we chose $\mathrm{d} B= \pm 2 \times 10^{-5}$.

For each elementary interval $\mathrm{d} t$, the sign of $\mathrm{d} B$ is picked arbitrarily. Thus, the $\mathrm{d} B$ signs in each $T_{k}$ are quite balanced, and so are along all the detection which lasts 201 elementary intervals: one can check that the sum of the quantities $\Sigma^{(k)} \operatorname{sign}(\mathrm{d} B)$ over all the process, i.e. over all the values of $k$, is equal to 3.

The formulas (A11) and (A10) obtained in the Appendix A for calculating $T_{k}$, are repeated below:

$$
T_{1}=12.84 \times 10^{-12} \mathrm{~s}, \text { i) } T_{k+1} \approx 0.917 T_{k} \text {. ii) }
$$

Beyond the column 11 the formula (40) cannot be used for arbitrary $\mathrm{d} B$ values in each $\mathrm{d} t$, because it would violate the inequality (45), i.e. would produce for $\left|a_{1}\right|^{2}$ values greater than 1 , or negative. Though, I succeeded to avoid obtaining $\left|\boldsymbol{a}_{1}\right|^{2}>1$ and $1-\left|\boldsymbol{a}_{1}\right|^{2}<0$ inside $T_{12}$, for some particular $\mathrm{d} B$ sets. As one can see, $\left|a_{1}\right|^{2}$ increased to a value very close to 1 .

Also, calculating $\langle\hat{N}\rangle$ at the end of $T_{12}$ with the relation (36i), there resulted $\langle\hat{N}\rangle \approx Y$ (the exact result was 40959.59). Thus, the $\mathrm{d} B$ sequence used in Table 1 , leads to the solution $(\mathbf{a}) .{ }^{10}$

Table 2 follows a different $\mathrm{d} B$ sequence with arbitrary signs, which leads to the prediction (b).

One can see that $\left|\boldsymbol{a}_{1}\right|^{2}$ practically vanishes, and so does $\langle\hat{N}\rangle$. The value obtained with the formula (36i) is 0.7 , while by the theorem 1 it should be much less. Though, given the very approximate calculi done here it makes no sense to investigate the difference.

Here, Equation (40) could not be used beyond the column 11. This equation makes the transit from the quantum to the classical domain, so, the fact that its validity stops seems to tell that the system of particles became classical.

Table 2. (a) Part 1. (b) Part 2.

(a)

\begin{tabular}{cccccccc}
\hline$k$ & 1 & 2 & 3 & 4 & 5 & 6 & 7 \\
\hline$T_{k}$ & 26 & 23 & 22 & 20 & 18 & 17 & 15 \\
$\Sigma^{(k)} \operatorname{sign}(\mathrm{d} B)$ & 0 & -1 & -2 & 0 & 4 & -1 & 1 \\
$Y_{k}$ & 20 & 40 & 80 & 160 & 320 & 640 & 1280 \\
$\left|\boldsymbol{a}_{1}\right|^{2}$ & 0.4 & 0.4 & 0.4 & 0.4 & 0.42 & 0.41 & 0.42 \\
\hline
\end{tabular}

(b)

\begin{tabular}{cccccc}
\hline$k$ & 8 & 9 & 10 & 11 & 12 \\
\hline$T_{k}$ & 14 & 13 & 12 & 11 & 10 \\
$\Sigma^{(k)} \operatorname{sign}(\mathrm{d} B)$ & 2 & 1 & -2 & -3 & 0 \\
$Y_{k}$ & 2560 & 5120 & 10,240 & 20,480 & 40,960 \\
$|\boldsymbol{a}|^{2}$ & 0.45 & 0.47 & 0.19 & $3.4 \times 10^{-5}$ & \\
\hline
\end{tabular}

${ }^{10}$ The calculi for the tables were performed with the utility EXCEL, and the reader may request a copy of them by e-mail. 
Therefore, in continuation the system should be treated with the Relation (41) which is purely classical. In support of that come the results in Table 1 and Table 2: before Equation (40) becomes invalid, $\left|\boldsymbol{a}_{1}\right|^{2}$ becomes practically equal to 1 , respectively zero. Substituting in the formula (36i) the final result for $\left|\boldsymbol{a}_{1}\right|^{2}$ in these tables, one gets $\langle\hat{N}\rangle \approx Y$, respectively $\langle\hat{N}\rangle \approx 0$ as requires the theorem 1.

\subsection{A Difficulty}

Not every $\mathrm{d} B$ sequence leads to a physical result, (a) or (b).

Even a different arrangement of the sequence of signs of $\mathrm{d} B$ within some interval $T_{k}$ in Table 1 and Table 2 may lead to a totally different final result. See for instance in Table 3: the part 1 is identical with the part 1 of Table 1, so, it is not repeated here. In the part 2 , the row $\Sigma^{(k)} \operatorname{sign}(\mathrm{d} B)$ is the same as the corresponding row in the part 2 of Table 1 . However, in the interval $T_{11}$ the sequence of signs of $\mathrm{d} B\{1,1,1,-1,-1,1,1,-1,1,-1,1\}$ was changed with the sequence $\{-1,1,1,-1,-1,1,1,-1,1,1,1\}$. As one can see, the resulting $\left|a_{1}\right|^{2}$ is completely different.

But, the essential fact is that after the process in the detector ended, there are no more PPC generations, though the result is neither $\left|\boldsymbol{a}_{1}\right|^{2} \approx 1$ as in Table 1 , nor $\left|\boldsymbol{a}_{1}\right|^{2} \approx 0$ as in Table 2. What is the output of the detector in such a trial? And what about the other two wave-packets, are they erased, or not?

Another problem with the response of the detector is that, in repeated trials, the physical results (a) and (b), should appear with the probabilities predicted by the initial wave-function, $\left|\boldsymbol{a}_{1}\right|^{2}$, respectively $1-\left|\boldsymbol{a}_{1}\right|^{2}$. So, it seems that the $\mathrm{dB}$ series have to be controlled by the initial wave-function. But, it is not clear how could that be possible, because the value of $\left|\boldsymbol{a}_{1}\right|^{2}$ changes in time, apparently there is no memory of $\left|\boldsymbol{a}_{1}\left(t_{0}\right)\right|^{2}$.

In connection with this problem, P. Pearle said in [12], that the probability of a solution of the normalized CSL equation is given by the norm of the non-normalized equation, not by the probability of the $\mathrm{d} B$ sequence which drove the evolution of the wave-function.

"Let $\mathrm{d} \Omega$ is the probability measure in the space of Brownian functions, and $w_{\Omega}$ be the particular Brownian function responsible for the evolution of the particular wavefunction $\psi_{\Omega}$, with squared norm

$$
N_{\Omega}^{2}(t)=\int \mathrm{d} x\left|\psi_{\Omega}(x, t)\right|^{2} 11
$$

... the probability that $\psi_{\Omega}$ lies in the ensemble ${ }^{12}$ is $N_{\Omega}^{2}(t)$, not $\mathrm{d} \Omega$.

... One expects that since $\mathrm{dw}$ ( or $\mathrm{d} B$ ) is as likely to fluctuate positively as negatively, the randomly fluctuating term ${ }^{13}$ will have only a modest effect

\footnotetext{
${ }^{11}$ Equation (8) in [12].

${ }^{12}$ In the ensemble of possible solutions.

${ }^{13}$ i.e. the term with $\mathrm{dw}$ or $\mathrm{dB}$ in the CSL equation-explanation of the present author.
} 
Table 3. Part 2.

\begin{tabular}{cccccc}
\hline$k$ & 8 & 9 & 10 & 11 & 12 \\
\hline$T_{k}$ & 14 & 13 & 12 & 11 & 10 \\
$\Sigma^{(k)} \operatorname{sign}(\mathrm{d} B)$ & -2 & -1 & 2 & 3 & 0 \\
$Y_{k}$ & 2560 & 5120 & 10,240 & 20,480 & 40,960 \\
$|\boldsymbol{a}|^{2}$ & 0.4 & 0.28 & 0.3 & 0.44 & 0.34 \\
\hline
\end{tabular}

for the majority of Brownian motions... Therefore, the norms of the wavefunctions evolving subject to these Brownian motions will decrease roughly exponentially...

On the other hand, for that minority of Brownian motions for which $\mathrm{dw}(\mathrm{x}, \mathrm{t})$, or a set of $\mathrm{d} B(\mathrm{z}, \mathrm{t}) \ldots$ happens to be of one sign significantly more frequently than of the other sign, the norm of the associated wavefunctions will grow..."14

However, Equation (40) in the present text has shown that the fluctuating sign of $\mathrm{d} B$ does not diminish the influence of the noise, since this influence is augmented by $Y$, which increases all the time.

S. Adler proposed as a solution to the above problem, a proof in [37] by which the CSL model predicts that the square variance of the measured operator, tends to regain its initial value if the measurement takes a very long time, and if the square of the measured operator commutes with the Hamiltonian or the Hamiltonian is negligible in comparison with the other terms in the SSE. These conditions are not fulfilled in the general case. In our case the Hamiltonian cannot be neglected, as it drives the creation of electron-ion pairs. It contains creation and annihilation operators, e.g. (34). Neither does it commute with the square of the measured operator, i.e. with $\hat{N}^{2}=\left(\begin{array}{llll}\hat{a}_{\mathrm{e}}^{?} & \hat{a}_{\text {ion }} & \hat{a}_{\mathrm{e}} & \hat{a}_{\text {ion }}\end{array}\right)^{2}$.

\subsection{Brief Discussion of a Related Work}

An analysis, related with the one presented in this text was done in [19]. A photon wave-function was supposed to contain two wave-packets, one of them hits a detector, the other doesn't. The configuration of the experiment is presented in Figure 7. The authors declared:

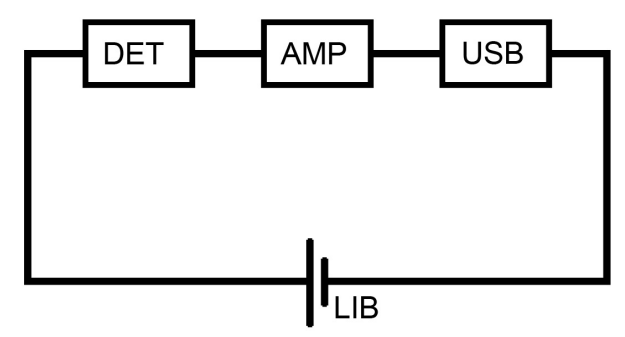

Figure 7. An alternative detection configuration. The circuit contains a Lithium-ion battery (LIB), a detector (DET), an amplifier (AMP), and a flash drive that records the signal.

${ }^{14}$ The wave-functions Pearle discussed are non-normalized. To the difference, we work here with the solutions of (34), which are normalized. 
"our essential assumption is that a measurement is completed by the time a permanent record of the event is made, that can be read out at a later time.

Each part of the setup has a different time scale, namely the detection time $t_{D}$, the amplification time $t_{A}$, and the recording time $t_{R}$. We thus identify the measurement time $t_{M}$ with the sum of these time scales."

The authors tried first to admit that the collapse is completed when the detector produces an output. That would have been logical, but they encountered a problem: for the value they assigned to the parameter $\lambda$ ( $\gamma$ in the present text) the collapse took too much time. On the other hand, as they showed in [36], this value depends on experiment-is not a universal constant. So, it's not clear why they didn't assign a greater value to that prameter.

Instead of that, they considered that the collapse ends in the battery. It seems non-logical. The battery is not a particle detector, all it does is to push current through a circuit. But, even before the battery, neither the amplifier nor a oscilloscope get as input a wave-function. One tunes the intensity of the input to these devices with a rheostat, not by a beam-splitter.

Besides, the amplification factor in a proportional ionization detector is not $10^{18}$ particles, but $10^{3}$ to $10^{4}$.

\section{Conclusions}

The CSL model for the measurement process of quantum systems has the advantage over different interpretations of the $\mathrm{QM}$, that it does not propose changes in the QM formalism as long as the studied object consists only in one or a few microscopic components. In this case the SSE reduces to the Schrödinger equation, as the additional terms are negligibly small. However, as the number of components of the system increases, these terms increase in magnitude becoming more significant than the Hamiltonian. In this way is simulated the passing from the microscopic to the macroscopic objects.

The CSL model is not an explanation of the collapse of the wave-function, as long as the nature of the noise incorporated in the SSE is not known. What it is clear, as explained in section 1, is that no classical field is the source of this noise. Thus, for the moment, this model represents a tool for investigating the collapse. In studying the noise, the test of the model on an experiment with a OPWF and a detector on only one of the wave-packets, is only a beginning. It already left open a problem, described in Subsection 5.4.

The configuration with detectors placed only on some of the wave-packets of a wave-function is an incomplete (or, non-maximal) test, in the sense defined in [38]. Despite the result obtained in the end of Table 1(b) that $\left|\boldsymbol{a}_{1}\right|^{2}$ becomes practically equal to 1 , one still cannot give a rigorous answer to the question asked in the beginning of section 1: when do the wave-packets $\left|\Psi_{2}\right\rangle$ and $\left|\Psi_{3}\right\rangle$ disappear?

The immediate answer of a reader would be, because Equation (35) is normalized, $\left|\boldsymbol{a}_{1}\right|^{2}=1$ entails instantly $\left|\boldsymbol{a}_{2}\right|^{2}+\left|\boldsymbol{a}_{3}\right|^{2}=0$. I prefer to postpone the an- 
swer until entanglements and relativistic cases are examined.

In this context I have to express criticism on a trend that seems to predominate at present: big efforts are invested in fitting Gaussian noises, non-white noise, finding the values of the parameter $\gamma$, while beyond the corner await the entanglements and the relativity, that may impose requirements that the CSL won't be able to satisfy.

If the CSL model won't be able to predict correctly the results of such experiments, it would remain just another model for local hidden variables.

\section{Conflicts of Interest}

The author declares no conflicts of interest regarding the publication of this paper.

\section{References}

[1] von Neumann, J. (1932) Mathematische Grundlagen der Quantenmechanik. Springer, Berlin.

[2] Lüders, G. (1950) Über die Zustandsänderung durch den Meßprozeß. Annalen der Physik, 443, 322-328. https://doi.org/10.1002/andp.19504430510

[3] de Broglie, L. (1926) Ondes et mouvements. Publisher Gauthier-Villars, Paris.

[4] Bohm, D. (1952) A Suggested Interpretation of the Quantum Theory in Terms of "Hidden" Variables. Part I and II. Physical Review, 85, 166-179. https://doi.org/10.1103/PhysRev.85.180

[5] Wechsler, S.D. (2019) The Wave-Particle Duality-Does the Concept of Particle Make Sense in Quantum Mechanics? Should We Ask the Second Quantization? J. of Journal of Quantum Information Science, 9, 155-170. https://doi.org/10.4236/jqis.2019.93008

[6] Hardy, L. (1992) On the Existence of Empty Waves in Quantum Theory. Physics Letters A, 167, 11-16. https://doi.org/10.1016/0375-9601(92)90618-V

[7] Griffiths, R.J. (2002) Consistent Quantum Theory. Cambridge University Press, Cambridge.

[8] Cramer, J.G. (1986) The Transactional Interpretation of the Quantum Mechanics. Reviews of Modern Physics, 58, 647-688.

[9] Ghirardi, G.-C., Rimini, A. and Weber, T. (1986) Unified Dynamics for Microscopic and Macroscopic Systems. Physical Review D, 34, 470. https://doi.org/10.1103/PhysRevD.34.470

[10] Ghirardi, G.-C., Pearle, P. and Rimini, A. (1990) Markov Processes in Hilbert Space and Continuous Spontaneous Localization of Systems of Identical Particles. Physical Review A: Atomic, Molecular, and Optical Physics, 42, 78-89. https://doi.org/10.1103/PhysRevA.42.78

[11] Gisin, N. (1984) Quantum Measurements and Stochastic Processes. Physical Review Letters, 52, 1657-1660. https://doi.org/10.1103/PhysRevLett.52.1657

[12] Pearle, P. (1989) Combining Stochastic Dynamical Statevector Reduction with Spontaneous Localization. Physical Review A, 39, 2277-2289. https://doi.org/10.1103/PhysRevA.39.2277

[13] Gisin, N. (1989) Stochastic Quantum Dynamics and Relativity. Helvetica Physica Acta, 62, 363-371. 
[14] Bassi, A. and Ghirardi, G.-C. (2003) Dynamical Reduction Models. Physics Reports, 379, 257. https://doi.org/10.1016/S0370-1573(03)00103-0

[15] Bassi, A., Lochan, K., Satin, S., Singh, T.P. and Ulbricht, H. (2013) Models of Wave-Function Collapse, Underlying Theories, and Experimental Tests. Reviews of Modern Physics, 85, 471-527. https://doi.org/10.1103/RevModPhys.85.471

[16] Bassi, A. and Salvetti, D.G.M. (2007) The Quantum Theory of Measurement within Dynamical Reduction Models. Journal of Physics A: Mathematical and Theoretical, 40, 9859-9876. https://doi.org/10.1088/1751-8113/40/32/011

[17] Bedingham, D.J. (2009) Dynamical State Reduction in an EPR Experiment. Journal of Physics A: Mathematical and Theoretical, 42, Article ID: 465301. https://doi.org/10.1088/1751-8113/42/46/465301

[18] Feynman, R.P. and Hibbs, A.R. (1965) Quantum Mechanics and Path Integral. McGraw-Hill Companies Inc., New York. Emended Edition Daniel F. Styer (2010) Emended Re-Publication Dover Publication, Inc., Mineola, New York.

[19] Adler, S.L. and Bassi, A. (2020) Minimum Measurement Time: Lower Bound on the Frequency Cutoff for Collapse Models. Journal of Physics A: Mathematical and Theoretical, 53, Article ID: 215302. https://doi.org/10.1088/1751-8121/ab8673

[20] Gisin, N. (2017) Collapse. What Else?

[21] Diósi, L. (1989) Models for Universal Reduction of Macroscopic Quantum Fluctuations. Physical Review A, 40, 1165-1174. https://doi.org/10.1103/PhysRevA.40.1165

[22] Diósi, L. (1987) A Universal Master Equation for the Gravitational Violation of Quantum Mechanics. Physics Letters A, 120, 377-381. https://doi.org/10.1016/0375-9601(87)90681-5

[23] Diósi, L. and Lukacs, B. (1987) In Favor of a Newtonian Quantum Gravity. Annalen der Physik, 499, 488-492. https://doi.org/10.1002/andp.19874990703

[24] Ghirardi, G.-C., Grassi, R. and Rimini, A. (1990) Continuous-Spontaneous-Reduction Model Involving Gravity. Physical Review A, 42, 1057-1064.

https://doi.org/10.1103/PhysRevA.42.1057

[25] Bassi, A. and Ghirardi, G.-C. (2002) Dynamical Reduction Models with General Gaussian Noises. Physical Review A, 65, Article ID: 042114. https://doi.org/10.1103/PhysRevA.65.042114

[26] Knoll, G.F. (2000) Radiation Detection and Measurement. 3rd Edition, John Willey and Sons Inc., Hoboken.

[27] Proportional Counters. https://www.orau.org/ptp/collection/proportional\%20counters/introprops.htm

[28] Diethorn, W. (1956) A Methane Proportional Counter System for Natural Radiocarbon Measurements. Thesis, Carnegie Inst. of Tech., Pittsburgh, Report Number NYO-6628.

[29] Milburn, G.J. (2010) Quantum Measurement and Control of Single Spins in Diamond. Science, 330, 1188-1189. https://doi.org/10.1126/science.1198299

[30] Elitzur, A.C. and Vaidman, L. (1993) Quantum Mechanical Interaction-Free Measurement. Foundations of Physics, 23, 987-997. https://doi.org/10.1007/BF00736012

[31] Sciarrino, F., Lombardi, E., Milani, G. and de Martini, F. (2002) Delayed-Choice Entanglement Swapping with Vacuum-One-Photon Quantum States. Physical Review $A, 66$, Article ID: 024309.

[32] Lombardi, E., Sciarrino, F., Popescu, S. and De Martini, F. (2001)Teleportation of Entangled States of a Vacuum-One Photon Qubit. https://arxiv.org/abs/quant-ph/0109160 
[33] Schellekens, M., Hoppeler, R., Perrin, A., Viana Gomes, J., Boiron, D., Aspect, A. and Westbrook, C.I. (2005) Hanbury Brown Twiss Effect for Ultracold Quantum Gases. Science, 310, 648-651. https://doi.org/10.1126/science.1118024

[34] Jeltes, T., McNamara, J.M., Hogervorst, W., Vassen, W., Krachmalnicoff, V., Schellekens, M., Perrin, A., Chang, H., Boiron, D., Aspect, A. and Westbrook, C.I. (2007) Comparison of the Hanbury Brown-Twiss Effect for Bosons and Fermions. Nature (Letters), 445, 402-405. https://doi.org/10.1038/nature05513

[35] MIT OpenCourseWare, Itô Calculus. From MIT 18.S096 Topics in Mathematics with Applications in Finance. Taught by Dr. Peter Kempthorne, Dr. Choongbum Lee, Dr. Vasily Strela, and Dr. Jake Xia in Fall 2013 (2013), Uploaded by MIT OpenCourseWare in Jan 2015.

https://ocw.mit.edu/courses/mathematics/18-s096-topics-in-mathematics-with-appl ications-in-finance-fall-2013/video-lectures/lecture-18-ito-calculus

[36] Adler, S.L. and Bassi, A. (2009) Is Quantum Theory Exact? Science, 325, 275-276. https://doi.org/10.1126/science. 1176858

[37] Adler, S. (2004) Proof of Reduction with Born Rule Probabilities. In: Adler, S., Ed., Quantum Theory as an Emergent Phenomenon, Cambridge University Press, Cambridge, Section 6.2. https://doi.org/10.1017/CBO9780511535277

[38] Peres, A. (1993) Maximal Quantum Tests. In: Quantum Theory: Concepts and Methods, Kluwer Academic Publishers, Dordrecht.

\author{
Abbreviations \\ APP $=$ amount of perturbed particles \\ $\mathrm{CSL}=$ continuous spontaneous localization \\ GPR $=$ Ghirardi, Pearle, Rimini \\ GRW $=$ Ghirardi, Rimini, Weber \\ LHS $=$ left hand side \\ OPWF = one particle wave-function \\ PPC = pair-producing collision \\ $\mathrm{QM}=$ quantum mechanics \\ RHS $=$ right hand side \\ SSE $=$ stochastic Schrödinger equation
}




\section{Appendix}

For each primary electron are liberated in the avalanche region a number $M$ of secondary electrons, number known under the name "multiplication factor"

$$
M=2^{\left|V_{a}-V_{1}\right| / \Delta V},
$$

where $V_{1}$ is the potential on the surface of the avalanche region, $V_{a}$ is the potential on the surface of the anode, and $\Delta V$ the potential fall along a path of an electron between two PPCs.

W. Diethorn calculated the potential fall $\left|V_{a}-V_{1}\right|$, and obtained section III.B part 3 in [28],

$$
\left|V_{a}-V_{1}\right|=\frac{V_{0}}{\ln (b / a)} \ln \left[\frac{V_{0}}{a p K \ln (b / a)}\right],
$$

where $V_{0}$ is the potential difference between the anode and cathode, $a$ is the radius of the wire, $b$ the internal radius of the cathode, $p$ the pressure, and $K$ is the minimal field intensity per unit of pressure, at which a secondary ionization can occur. ${ }^{15}$ For the mixture of gases mentioned in Subsection 5.3, table 6.1 in chapter 6 section III.A of [26] indicates $\Delta V=31.4 \mathrm{~V}$ and $K=36600 \mathrm{~V} / \mathrm{cm} \cdot \mathrm{atm}$, so that with $a=0.008 \mathrm{~cm}, b=1 \mathrm{~cm}, V_{0}=1750 \mathrm{~V}$, and $p=0.4 \mathrm{~atm}$, one obtains $\left|V_{a}-V_{1}\right| \approx 400.5 \mathrm{~V}$. Using this in (A1) there results, under the assumption $\left.{ }^{*}\right)$ in section 5.1, that the number of PPC generations in the avalanche is 12. (In fact, $\left|V_{a}-V_{1}\right| / \Delta V \approx 12.75$ i.e. the last PPC generation does not take place on the surface of the anode.)

With the numerical data mentioned above there results a multiplication factor $M=4096$.

In the cylindrical geometry the relation between the potential at two points, at distances $r_{1}$, respectively $r_{2}$, from the central axis, is

$$
\ln \left(r_{2} / r_{1}\right)=-\frac{V_{2}-V_{1}}{V_{0}} \ln (b / a) \text {. }
$$

Setting $r_{2}=b, \quad r_{1}$ equal to the radius of the avalanche region, and $V_{1}=1750-400.5 \approx 1349.5 \mathrm{~V}$, one gets $r_{1}=0.024 \mathrm{~cm}$. From this radius inwards, we can make iteratively a rough evaluation of the radius of each PPCs region.

$$
r_{k+1} / r_{k}=\exp \left[\frac{-\Delta V}{V_{0}} \ln (b / a)\right]=\exp \left[\frac{-31.4}{1750} \times 4.8283\right] \approx 0.917 .
$$

The duration of the interval of flight between two PPCs can be calculated with the equality $\mathrm{d} r=v \mathrm{~d} t$. As we will see below, the energies at which an electron is accelerated in the avalanche region render its wavelength so small that the movement of the wave-packet can be calculated with the classical kinematics. So, for the velocity $v(r)$ in the region $\left[r_{k}, r_{k+1}\right]$ we can use the formula

$$
v(r)=\sqrt{2\left(V_{r}-V_{r_{k}}\right) e / m_{0}},
$$

\footnotetext{
${ }^{15}$ The notation of the parameters here differs from that in [28], it is the same as in [26].
} 
$e$ being the elementary charge, $m_{0}$ the electron mass, and $r<r_{k}$.

With the help of (A3) we can translate the difference of potentials in (A5) into ratios of radiuses

$$
v(r)=\sqrt{-2 \frac{V_{0} e}{\ln (b / a) m_{0}} \ln \left(r / r_{k}\right)} \approx 1.077 \times 10^{9} \sqrt{-\ln \left(r / r_{k}\right)},
$$

the result being in units of $\mathrm{cm} / \mathrm{s}$. Thus, we get the equation

$$
\mathrm{d} r / \sqrt{-\ln \left(r / r_{k}\right)}=1.077 \times 10^{9} \mathrm{~d} t .
$$

This equation is difficult to integrate, so, let's try to simplify it. From (A4) one can see that $r$ and $r_{k}$ are very close, therefore we can develop $\ln \left(r / r_{k}\right)$ in Taylor series of the quantity $r_{k}-r=\varepsilon$. Retaining only the first order of smallness, $-\ln \left[\left(r_{k}-\varepsilon\right) / r_{k}\right] \approx \varepsilon / r_{k}$. Thus, we obtain the differential equation

$$
\left.1.077 \times 10^{9} \mathrm{~d} t=\frac{-\mathrm{d} x}{\sqrt{1-x}} r_{k}, \text { i) } \quad x=r / r_{k} . \text { ii }\right)
$$

where the radius is in $\mathrm{cm}$, and the time in seconds.

Integrating the RHS of (A8i) from $r_{k}$ to $r_{k+1}$, and the LHS from the time the electron reaches to radius $r_{k}$ to the time it reaches $r_{k+1}$, interval we denote by $T_{k}$, there results

$$
1.077 \times 10^{9} T_{k}=2 r_{k} \sqrt{1-r_{k+1} / r_{k}} .
$$

From this equation and from (A4) it's obvious that the following recursive relation emerges

$$
T_{k+1} / T_{k}=r_{k+1} / r_{k} \approx 0.917 .
$$

However, we have first to calculate $T_{1}$. Setting in (A9) $k=1$, using $r_{1}=0.024$ found above, one obtains

$$
T_{1}=12.84 \times 10^{-12} \mathrm{~s} .
$$

